\title{
LA ESTELA DE IXIMCHE' EN EL CONTEXTO DE LA REVITALIZACIÓN LINGÜÍSTICA Y LA RECUPERACIÓN JEROGLÍFICA EN LAS COMUNIDADES MAYAS DE GUATEMALA
}

\author{
Mallory E. Matsumoto \\ Departamento de Antropología de las Américas, \\ Universität Bonn
}

\begin{abstract}
Resumen: Los avances en el estudio de la escritura jeroglífica maya constituyen un componente dinámico del movimiento maya en Guatemala y ofrecen una gran oportunidad de observar la recuperación de una escritura dormida. Este estudio analiza el texto jeroglífico en la estela moderna recién erigida en Iximche', Chimaltenango, Guatemala, y propone que ésta manifiesta varias estrategias de la revitalización de lenguas mayas modernas y de la construcción de una identidad panmaya. El aprendizaje y el uso activo de la escritura antigua en el presente, en éste y otros medios, reflejan la dinámica entre la identidad panmaya y la identidad peculiar del pueblo kaqchikel. Además, por retomar una tradición monumental y de escritura antigua, afirma el vínculo cultural sentido por el pueblo maya contemporáneo con su pasado precolombino. Con el estudio de esta recuperación se puede ampliar también nuestro entendimiento del papel de la escritura en cuanto a expresar, reinterpretar y desarrollar tradiciones e identidades.
\end{abstract}

Palabras clave: escritura maya jeroglífica, estela de Iximche', recuperación linguiística, identidad panmaya, kaqchikel.

AвSTRACT: The revival of the Mayan hieroglyphic script constitutes a dynamic component of the Maya Movement in Guatemala and offers a rare opportunity to observe the reclamation of a dormant writing system. This study analyzes the hieroglyphic text on the recently erected stela at Iximche', Chimaltenango, Guatemala, and proposes that it manifests various strategies used in revitalizing the modern Mayan languages and in the construction of a Pan-Maya identity. The acquisition and active use of the ancient script in the present in this and other media reflects the dynamic between the Pan-Maya identity and the distinctive identity of the Kaqchikel people. Furthermore, by reasserting an ancient monumental and scribal tradition, it affirms the cultural link experienced by the Maya peoples with the pre-Columbian past. The study of the revived script thus may also enhance our understanding of the role of writing in expressing, reinterpreting, and developing traditions and identities.

KeYwords: Mayan hieroglyphic script, Iximche' stela, language revival, Pan-Maya identity, Kaqchikel.

RECEPCIÓN: 18 de febrero de 2014.

ACEPTACIÓN: 29 de mayo de 2014. 



\title{
LA ESTELA DE IXIMCHE' EN EL CONTEXTO DE LA REVITALIZACIÓN LINGÜÍSTICA Y LA RECUPERACIÓN JEROGLÍFICA EN LAS COMUNIDADES MAYAS DE GUATEMALA
}

\author{
Mallory E. Matsumoto \\ Departamento de Antropología de las Américas, \\ Universität Bonn
}

El movimiento maya y la revitalización de las lenguas mayas en Guatemala

El movimiento maya, de tipo cultural y político, surge en Guatemala hacia 1980, y tiene como objetivo principal la revaloración de las culturas mayas y el acceso de las comunidades mayas a los derechos políticos que se les han negado desde la época colonial (Barrett, 2008: 277; Brown, 1998a: 168-169; Cojtí Cuxil, 1996: 29-36; England, 2002: 34; Fischer, 1996: 63-64; Montejo, 2002: 144-145, 2005). En su aspecto cultural, el movimiento reafirma y promueve los que considera rasgos culturales sobresalientes, como el traje, el calendario, la espiritualidad y el idioma (Brown, 1996: 174; England, 2002: 34; Normark, 2004: 131; Otzoy, 1996: 147). Un componente fundamental del movimiento es la construcción y promoción de una identidad panmaya para unir a las comunidades en Guatemala y así aumentar su vivacidad cultural y poder político (Brown, 1998a: 156; Cojtí Cuxil, 1996: 41-43; Montejo, 2005: 16-36; Sturm, 1996: 115; p. ej. Maxwell, 1996: 205-206). ${ }^{1}$

Este trabajo se enfoca principalmente en la identidad a nivel de la colectividad, y no en la identidad individual, porque los fenómenos de la recuperación jeroglífica y la estela de Iximche' están vinculados, sobre todo, con los procesos de establecer una identidad colectiva, definida como el sentido de pertenencia a un colectivo y la estrategia dinámica de asociarse con un grupo que comparte características que se consideran determinantes (Díaz-Andreu y Lucy, 2005: 1-2; Unseth, 2005).

El concepto de una identidad panmaya se basa en gran parte en el diálogo arqueológico, antropológico y lingüístico sobre el pueblo maya y también en la

\footnotetext{
${ }^{1}$ La abreviatura "p. ej." (por ejemplo) que aparece de aquí en adelante remite a textos que dan cuenta de manera puntal — v. g. a través de estudios etnográficos- de los supuestos o planteamientos teóricos generales a los que aluden los otros autores citados.
} 
historia de opresión que han compartido todas las comunidades en los últimos siglos (Brown, 1998a: 168-169, 1998b: 44-45; Montejo, 2005: 17-22, 142; Sturm, 1996: 119; p. ej. Cojtí Cuxil, 1996; Grube y Fahsen, 2002: 233; Normark, 2004). Aunque la diversidad cultural y lingüística que caracteriza al pueblo contemporáneo seguramente se manifestaba entre las comunidades precolombinas (p. ej. Montejo, 2002, 2005: 22-27), muchos estudios sobre los mayas antiguos hacen hincapié en la evidencia de los rasgos culturales comunes, como los idiomas (p. ej. Campbell y Kaufman, 1985; Law, 2013) o la arquitectura (p. ej. Christie, 2010; Houston, 1998). La imagen de una herencia compartida en el presente les sirve a las comunidades actuales para construir una identidad compartida y un sentido de unidad cultural y política (Brown, 1998a: 168-169, 1998b: 44-45; Montejo, 2005: 17-22). Este fenómeno se manifiesta, por ejemplo, con la creación de una bandera maya como símbolo de esta identidad, aunque nunca existió un "reino" maya unido ni siquiera en la época precolombina (Martin y Grube, 2008: 7).

Hay que enfatizar que, a diferencia de lo que ocurre en el caso de la escritura jeroglífica, en lo que respecta a la revitalización de las lenguas modernas que está teniendo lugar en el área maya de Guatemala hoy en día no se trata de la recuperación de lenguas que ya se dejaron de usar y que están "dormidas" (Amery, 2001: 148), si no que se refiere más bien al rescate de idiomas que se están perdiendo por el desplazamiento linguí́stico debido al empleo del castellano, sobre todo en las generaciones más jóvenes (Barrett, 2008: 276-278; véase Fishman, 2011: 223-224; Tsunoda, 2005: 200-202).

En literatura, la revitalización linguística tiene varios sentidos que se diferencian según el estatus del idioma que se está revitalizando. En general, el término suele hacer referencia al proceso de revivificar y reforzar un idioma que ya cayó en desuso o está en peligro de extinción, porque la comunidad de hablantes es reducida o inexistente (Tsunoda, 2005: 168; England, 1998: 99). Este proceso puede incluir, entre otras estrategias, la planificación linguística y el establecimiento de una ortografía, si el idioma no cuenta con una tradición escrita. Tiene como meta el mantenimiento y la expansión de la comunidad de hablantes (Tsunoda, 2005: 171214). En el caso de lenguas que cayeron en desuso, como la escritura jeroglífica maya y el hebreo (Houston, 2008; Zuckermann, 2009), el empleo del término "revitalización" remite más bien a un proceso de recuperación mediante el cual se intenta restaurar el idioma en una comunidad que ya no lo utiliza (Tsunoda, 2005: 168). De esta manera, en este trabajo el término "revitalización" hace referencia únicamente al caso de los idiomas mayas contemporáneos, que están en vías de extinción pero cuentan todavía con una comunidad de hablantes. En cambio, se deberá discutir sobre el proceso de revivificar la escritura jeroglífica como proceso de "recuperación" linguística, que consiste en revivir y reintroducir el sistema de escritura basándose en fuentes históricas ${ }^{2}$ (Tsunoda, 2005: 168).

\footnotetext{
2 Al transliterar, transcribir y traducir los textos jeroglíficos, sigo las normas explicadas por Kettunen y Helmke (2011: 14-16). La ortografía empleada para el idioma kaqchikel contemporáneo respeta
} 
Es de destacar que una lengua no se mantiene constante a través de estos procesos, sino que se sigue desarrollando bajo la influencia del contexto linguístico, cultural y sociopolítico de su revitalización o recuperación, el cual condiciona, por ejemplo, la ideología linguística y la percepción del valor del idioma, los motivos para retomar la lengua perdida o ya dormida, y los recursos puestos a disposición de los estudiantes para lograr este objetivo (Spolsky, 1991: 138140). Además, los otros idiomas que son corrientes entre los participantes en la revitalización o recuperación pueden influir en la estructura linguística y en el lexicón de la lengua revivificada (Amery, 2001: 182-184; Grenoble, 2013: 809; p. ej. Barrett, 2008; Jones, 1998: 358). Por ejemplo, las lenguas maternas europeas de los líderes de la recuperación del hebreo antiguo en Israel condicionaron la estructura y los patrones de uso del idioma, por lo cual el hebreo contemporáneo se considera como una lengua híbrida (Zuckermann, 2009). Las lenguas revitalizadas también varían debido a la influencia de las decisiones que toman los participantes en el proceso, como, por ejemplo, al elegir formas estandarizadas (Maxwell, 1996). La revitalización y la recuperación no deben ser, pues, entendidas como un resurgimiento o como la preservación de una lengua, ni en la forma original o en una equivalente, ni en la forma que se pretende revitalizar o recuperar. Se trata más bien del desarrollo y del establecimiento de una forma de la lengua adaptada a su nuevo contexto linguístico, cultural y sociopolítico (véase p. ej. Amery, 2001: 141-142).

La revitalización linguiística de los idiomas contemporáneos representa un enfoque principal de la revaloración de las culturas mayas en Guatemala debido al papel central de la lengua en las comunidades como componente básico de la identidad (Brown, 1996: 172-173, 1998a: 158; England, 1996: 178, 2003: 733-737; Fischer, 1996: 57, 65; French, 2010: 32-39; Maxwell, 1996: 197-199). Tanto los idiomas mismos como el diálogo sobre una historia de opresión linguística compartida aportan la creación de una identidad común y un sentimiento de solidaridad entre las comunidades mayas (French, 2010: 33). Los líderes de este movimiento tienen como objetivo no sólo el mantenimiento y la revaluación de sus idiomas para combatir el desplazamiento linguiístico a favor del castellano, sino también la expansión de los campos de uso de las lenguas (Brown, 1998a: 159; Cojtí Cuxil, 1996: 36-38; England, 1996: 178-179, 2003: 733; véase Fishman, 2011).

Una estrategia para lograr esto último ha sido, por ejemplo, la creación de neologismos mayas para indicar conceptos modernos (p. ej. en kaqchikel kematz'ib', "computadora", una palabra compuesta de las raíces kem, "tejer", y $t z$ 'ib', "escritura") en lugar de usar un préstamo linguíístico del castellano (Barrett, 2008: 281-282; Brown, 1996: 174; French, 2010: 32-33, 35; Maxwell, 1996: 201-202). También anhelan modificar la ideología linguíística, ya que, desde la época colonial, las lenguas mayas se relacionan de manera peyorativa con fenómenos como

las normas de la Academia de las Lenguas Mayas de Guatemala (apud Brown et al., 2006: 8-11). Todas las imágenes son de mi autoría. 
la pobreza, la falta de estudios y la ignorancia (Barrett, 2008: 276-277; England, 2003: 736-737). Para poder detener e invertir el proceso del desplazamiento linguístico, se considera necesario que la actitud de los hablantes nativos frente a sus propios idiomas cambie (Bell, 2013; Grenoble, 2013: 797).

A causa de la diversidad en la familia de lenguas y la existencia de muchas variantes en cada idioma, la estandarización de las lenguas mayas constituye parte fundamental de la revitalización linguiística, además de que apoya la construcción de una identidad panmaya por facilitar la comunicación entre las diversas comunidades (Barrett, 2008: 279; England, 1996: 179; French, 2010: 35), y se aplica sobre todo a la forma escrita. Es probable que se pueda hablar de un cierto grado de estandarización del idioma escrito en la época precolombina, pero, si hubiese sido éste el caso, ésta desapareció junto con las habilidades de lectoescritura después de la conquista. Esta situación, y la política colonial de separación geográfica de las poblaciones, motivaron una diversificación aún mayor dentro de la familia de lenguas mayas (England, 1996: 194, 2003: 739; Maxwell, 1996: 199200). Los líderes de la revitalización linguiística, a los cuales pertenecen muchos expertos del pueblo kaqchikel (Barrett, 2008: 281; Maxwell, 1996: 197-198), piensan que la estandarización es necesaria para detener la diversificación dialectal y apoyar la identidad panmaya (p. ej. England, 2003: 737). Con ese fin, en octubre de 1986 se fundó la Academia de las Lenguas Mayas de Guatemala (ALMG), cuyo primer logro fue el establecimiento de un alfabeto único y oficial para todos los idiomas mayas en Guatemala (Brown, 1998a: 157-158; England, 1996: 183-184).

A pesar de la diversidad linguística entre las comunidades mayas, y tal vez a causa de ella, en pocos casos existe una sola variante entre las muchas de una lengua que buena parte de la comunidad de hablantes considere como prestigiosa (England, 1996: 182). Al contrario, la mayoría de los hablantes considera su propia variante local como la correcta, lo que puede crear conflictos durante el proceso de la estandarización linguística, por ejemplo, a la hora de elegir una única forma estándar para las muchas variantes (England, 1996: 179, 185; 2003 : 736-739; French, 2010: 65-76). Debido en parte a este fenómeno, los expertos que encabezan el proceso de estandarización tienden a establecer una forma unida y estándar, combinando varias maneras o reconociendo múltiples formas existentes como sinónimos en lugar de estandarizar una sola, tomada de una variante elegida (England, 1996: 187; 2003: 736).

Otro factor significativo en la elección de formas estándares ha sido la historia linguística. En la estandarización de las lenguas mayas, se suelen utilizar los modelos ya conocidos por los textos de la época colonial o a través de reconstrucciones linguísticas (Barrett, 2008; England, 1996: 187-188; Fischer, 1996: 65; French, 2010: 33-34). Por ejemplo, se propuso establecer -uma como la forma estándar del sustantivo relacional "por, porque" en kaqchikel, aunque este elemento aparece como -oma en la mayoría de las variantes kaqchikeles (Brown et al., 2006: 157; Maxwell y Little, 2006: 22). A pesar de ser una forma minoritaria entre los hablantes de kaqchikel, se argumentaba que -uma tiene la ventaja de aparecer, junto 
con la variante -uma(a)l, en las crónicas kaqchikeles de los siglos xvI y xvI, y además de semejar la forma -umaal que todavía se encuentra en el idioma k'ichee', el cual está muy estrechamente relacionado con el kaqchikel (Maxwell y Hill, 2006, Parte II: 2, 21; Maxwell y Little, 2006: 22). En general, se consideran tales formas con atestación histórica más pura o válida, porque pertenecen a una época en la que la influencia del castellano en el kaqchikel era restringida o quizás completamente inexistente (Brown, 1996: 175, 1998b: 45; England, 1996: 189-190; Maxwell, 1996: 201). Además, la forma kaqchikel -uma, por parecerse tanto a la forma k'ichee' -uma(a)l, cumple con otro criterio importante en la estandarización, es decir, la semejanza con las formas en otras lenguas, la cual sirve para facilitar la comunicación entre las comunidades del pueblo maya (England, 1996: 187).

Una característica clave del proceso de estandarización, como del movimiento maya en sí, es el papel central de la autodeterminación del pueblo para tomar las decisiones sobre su propio destino cultural y linguístico (p. ej. Fischer, 1996: 64). Desde el comienzo, por ejemplo, del establecimiento del alfabeto oficial para las lenguas mayas en Guatemala, los líderes mayas consultaron con linguiistas y otros expertos extranjeros, pero no les autorizaron a participar en la toma de decisiones, lo cual sólo se le permitió a los expertos mayas. Esto estableció un patrón en el proceso de estandarización que contrasta fuertemente con el patrón anterior de someterse a las decisiones de extranjeros (Brown, 1996: 174, 1998a: 156-157; England, 1996: 183-184). De tal modo, la estandarización contribuye al objetivo general del movimiento de establecer la autodeterminación y el autogobierno del pueblo maya, cuyo destino cultural, económico y sociopolítico ha sido guiado en gran medida por personas no mayas desde hace muchos siglos (Montejo, 2005: 173-175).

\section{La recuperación de la escritura jeroglífica maya}

Junto con la revitalización de las lenguas modernas, la recuperación de la escritura jeroglífica está teniendo lugar en Guatemala, donde se había dejado de usar y de la cual se ha perdido el conocimiento en las generaciones posteriores a la Conquista (England, 1996: 181; Houston, 2008). Este proceso corresponde al rescate de una lengua dormida, con base en fuentes históricas y académicas, al contrario de la revitalización de las lenguas modernas (Amery, 2001: 141, 148; Fishman, 2011: 222-223; Tsunoda, 2005: 168, 200-202). Sin embargo, se diferencia del proceso más común de recuperar un idioma, por ejemplo el hebreo (Zuckermann, 2009) o el manés (Wilson, 2009), porque se enfoca en el sistema de escritura, y no la lengua hablada. ${ }^{3}$ Aunque la ortografía suele jugar un papel importante en la revitalización o recuperación de lenguas habladas, hacerlo con un sistema de

\footnotetext{
${ }^{3}$ Acerca de la recuperación de una lengua hablada con base en documentos históricos, cf. Amery, 2001.
} 
escritura es un fenómeno poco común, por lo cual el contexto actual de los mayas en Guatemala presenta una oportunidad excepcional de apreciar tal proceso y sus resultados (Unseth, 2005: 31). ${ }^{4}$

Las diferencias fundamentales entre la lengua hablada y la escrita son varias e incluyen el proceso de adquisición, que ocurre, en el caso del idioma hablado, sin intención alguna, normalmente durante la niñez y bajo la influencia del habla de los adultos. En cambio, la adquisición de la escritura siempre es un proceso consciente y casi siempre sigue a la adquisición de la forma hablada. ${ }^{5}$ Además, se distinguen por los contextos de uso, que son en general más interactivos y sociales con el habla que con la escritura. La durabilidad es una función fundamental en el desarrollo y en el uso de la escritura, pero no caracteriza la gran mayoría de los actos de habla y sólo se ha extendido en las últimas generaciones gracias al desarrollo tecnológico. La espontaneidad también suele caracterizar los actos de habla, mientras que lo escrito por lo común toma mucho más tiempo, permitiendo una mayor reflexión antes de reproducir el habla, lo que hace posible su corrección y mejoramiento antes de la publicación (Chafe, 1985: 105; Scilar-Cabral, 2012: 40-44). La recuperación de las dos formas de la lengua se diferencia por el hecho de que el cerebro ni percibe ni procesa el habla y la escritura de la misma manera (Scilar-Cabral, 2012: 41-44; Warren, 2013: 99-115).

Estas diferencias cruciales entre el habla y la escritura también deben influir en el proceso de recuperarlas. La revitalización o la recuperación de lenguas habladas es un tema muy discutido en la lingüística actual (King, 2001: 5-12; Tsunoda, 2005: 168-169; p. ej. Wilson, 2009; Zuckermann, 2009; Zuckermann y Walsh, 2011). En cambio, todavía no existe una base similar de material de consulta sobre la revitalización o la recuperación de una escritura, en principio porque hay muy pocos precedentes para ello; además, muchas, si no la mayor parte, de las lenguas que se han revitalizado o recuperado, carecían de un sistema indígena de escritura (Amery, 2001: 176-178; Grenoble, 2013: 799-800; Tsunoda, 2005: 188-189). La recuperación de la escritura jeroglífica maya es una oportunidad única de observar el proceso de recuperación de un sistema de escritura, sin

\footnotetext{
${ }^{4}$ Uno de los pocos precedentes para la reintroducción de una escritura que se había dejado de usar es lo registrado con el sistema de escritura mongol, que coexiste ahora en Mongolia en una situación de digrafía con el alfabeto cirílico, la cual se revivificó sólo unas seis décadas después de su desplazamiento frente a la escritura cirílica promovida por el gobierno soviético (Grivelet, 2001: 76-78), a diferencia de la situación actual en Guatemala. Además, después de estar oficialmente remplazada, la escritura mongola no se había perdido tanto como en el caso de la escritura jeroglífica maya; se había conservado en universidades, entre los ancianos en la población, y también como escritura activa en la región de Mongolia interior (Grivelet, id.). En consecuencia, en Mongolia no se trata tanto de la recuperación de una escritura dormida, sino de la revitalización de una escritura tradicional después de un periodo de represión. Otro ejemplo de la recuperación de un sistema de escritura dormido es la reintroducción de la escritura tifinagh entre los bereberes en África del Norte, un movimiento politizado y controvertido que no ha tenido mucho éxito (Pouessel, 2008; Savage, 2008: 6-7; Zouhir, 2014). Como la escritura mongola, este sistema de escritura también parece haber sido restringido a contextos más limitados en el pasado que la escritura jeroglífica maya.

${ }^{5}$ Para el caso específico de individuos sin audición, cf. Steinberg y Sciarini, 2006: 56-64.
} 
recuperar la(s) lengua(s) hablada(s) anteriormente asociada(s) a esta forma escrita, y adaptarlo a su nuevo contexto linguiístico, cultural y sociopolítico.

El sistema de escritura jeroglífico maya se califica como logosilábico, consiste principalmente en logogramas, los cuales representan una palabra o un morfema, así como silabogramas, que dan cuenta de una sílaba (Kettunen y Helmke, 2011: 8). Tiene un orden de lectura básico de izquierda a derecha y de arriba abajo. Los glifos se organizan individualmente en bloques cuadrados dentro del texto. Muchos de los textos precolombinos consisten en columnas de bloques jeroglíficos, los que se leen en columnas dobles, pero también hay numerosas excepciones de textos organizados en bloques individuales, hileras horizontales u otras estructuras atípicas (Kettunen y Helmke, 2011: 17; p. ej. Matsumoto, 2013; Robicsek, 1975). Salvo unos pocos signos diacríticos y determinativos semánticos, todos los jeroglíficos en el corpus precolombino, tanto los logogramas como los glifos silábicos, tienen por lo menos un valor fonético basado en la estructura silábica básica de consonante-vocal (Kettunen y Helmke, 2011: 21-22; Mora-Marín, 2003a: 197). Existía la posibilidad de escribir muchas palabras con logogramas y/o con silabogramas, y la elección de una de estas alternativas varía según el texto y a veces de acuerdo al bloque glífico dentro del mismo (Mora-Marín, 2003a: 198). ${ }^{6}$ Se supone que el idioma grabado en los textos jeroglíficos precolombinos, el que se suele llamar "maya clásico", era un idioma ch'olano o en algunos textos una forma del yukateko, pero la(s) lengua(s) empleada(s) en los glifos es todavía un tema controvertido (Houston, Robertson y Stuart, 2000, 2001; Kettunen y Helmke, 2011: 13; Macri y Looper, 2003: 8-9; Macri y Vail, 2009: 7-8; Mora-Marín, 2003a, 2009; Sturm, 1996: 119). También se está discutiendo acerca de qué tan difundido estaba el alfabeto jeroglífico y qué tipo(s) de alfabetismo había en las poblaciones mayas precolombinas. Sin embargo, es probable que este conocimiento, sobre todo la habilidad de interpretar un texto glífico linguiística y literalmente, estuviera relativamente restringido (Grube y Fahsen, 2002: 233; Houston, 1994: 40).

Lacadena (1995) observa en los textos precolombinos evidencias de una recuperación de variantes individuales de glifos antiguos a través de la historia de la escritura, tal vez como un intento por vincularse simbólicamente de forma gráfica con los textos históricos.

Pero la recuperación de la escritura hoy en día no sólo es mucho más amplia que este fenómeno del pasado, sino que además falta el desarrollo continuo de la escritura. Es decir, el proceso actual de recuperar el sistema de escritura jeroglífica se caracteriza por una distancia, tanto temporal como intelectual, entre su forma precolombina y la moderna, ya que la transmisión actual de la escritura sólo es

\footnotetext{
${ }^{6}$ Por ejemplo, se escribe Pakal en el nombre del líder de Palenque K’inich Janab، Pakal con el logograma PAKAL con complemento fonético la en el bloque glífico R11 (PAKAL-la) en el tablero este del Templo de las Inscripciones en Palenque, pero con los glifos silábicos pa-ka-la en bloque glífico S13 del mismo texto (véase Schele, 2000: 152).
} 
posible gracias a los resultados de la investigación epigráfica y linguística de las últimas décadas, no a una tradición escritural continua del pasado precolombino. Durante el periodo posterior a la llegada de los europeos, los glifos dejaron de usarse (England, 1996: 181; Houston, 2008). Más tarde llamaron la atención de unos viajeros extranjeros, e incluso algunos de ellos intentaron interpretarlos. Pero sólo a partir de la segunda mitad del siglo xx se ha podido avanzar a buen paso en el desciframiento de la escritura (Krochock, 2002: 267- 272; Houston, Chinchilla y Stuart, 2001: 10-13, 21; Sturm, 1996: 117). Es de recordar que fueron europeos y estadounidenses, los que llevaron a cabo los primeros trabajos del desciframiento (Grube y Fahsen, 2002: 217-218; Houston, Mazariegos y Stuart, 2001: 10-13; Krochock, 2002: 267-272; Sturm 1996: 126), y desde hace pocos años, epigrafistas del mismo pueblo maya se han juntado a este proyecto (Sturm, 1996: 126-128).

Pero la historia moderna de la investigación y del aprendizaje de la escritura antigua es más larga que la de su uso activo. Hace ya algunas décadas, académicos europeos y estadounidenses, y ahora también más y más mayas, dan talleres de epigrafía para gente interesada en las mismas comunidades mayahablantes (Grube y Fahsen, 2002; Schele y Grube, 1996). El objetivo no sólo es compartir el conocimiento de la escritura maya antigua o motivar a miembros del pueblo maya a investigar su pasado precolombino, sino también despertar el interés de las comunidades por su propia historia (Schele y Grube, 1996: 136-139).

El primer uso regular de la escritura, en su forma recuperada, se aprecia en las publicaciones de la editorial guatemalteca Cholsamaj, en las cuales se empezó a escribir la fecha de publicación y los números de página con glifos en el año 1994 (Rodríguez Guaján, com. pers., 2013; Sturm, 1996: 120-125). En las últimas décadas se han utilizado los glifos en contextos siempre más diversos, como en vasijas cerámicas, libros, murales, cartas privadas y también en medios nuevos como páginas web (Brown, 1998a: 157; Sturm, 1996: 122-126; p. ej. Grube y Fahsen, 2002: 233, fig. 13.4; MAM, 2013a, 2014). Además se han erigido varias estelas o monumentos de piedra situados de forma independiente, siguiendo así el ejemplo de las estelas precolombinas. Sé que existen por lo menos 13 de tales monumentos en Guatemala, además de tres en México (Cojtí Cuxil, 2013; Rodríguez Guaján, com. pers., 2013; MAM, 2013b-c; Mucia Batz, 2012). ${ }^{7}$

Al trasladarse la escritura antigua al presente, ésta se transforma en un símbolo de las culturas mayas históricas y contemporáneas, y así contribuye a la

${ }^{7}$ De éstas, 10 se erigieron para conmemorar el fin del $13^{\circ}$ bak'tun: dos en México (Mérida y Valladolid, Yucatán) y las restantes en Guatemala (en el Departamento de Chimaltenango: Iximché, Tecpán, Mixco Viejo [Chwa Nima’ab’aj], Patzicía, San Martín Jilotepeque y Patzún; en el de Totonicapán, Momostenango; en el de Sololá, Tzununya, de San Pedro la Laguna, y en Poptún, departamento de Petén). Seis más se construyeron para otras ocasiones: tres en Cobán, Alta Verapaz (dos en el Instituto Magisterio de Preprimaria Bilinguie), una en el Colegio Maya Poqomam de Palín, Escuintla, y una más en San Juan Ostuncalco, departamento de Quetzaltenango. La sexta fue en México, en la escuela agrícola U Yit's Ca'an en Maní, en Yucatán. 
creación de una identidad panmaya compartida (Brown, 1998a: 157; Grube y Fahsen, 2002: 233; Sturm, 1996: 117). Incluso si sólo una minoría del pueblo maya puede leer y entender los textos jeroglíficos linguísticamente, el significado cultural de los glifos, como manifestación gráfica de una existencia y colectividad maya, se ha generalizado (Grube y Fahsen, 2002: 233-234; Sturm, 1996: 118-125). Hoy en día se usan sobre todo logogramas del corpus jeroglífico, porque éstos comunican un significado determinado que los miembros de todas las comunidades linguísticas pueden entender, sin necesidad de saber el valor fonético del logograma (Sturm, 1996: 120). Para los líderes y los participantes en el movimiento maya, la aplicación de la escritura jeroglífica a contextos modernos ilustra tanto el orgullo que se siente por su ascendencia, como la lucha contemporánea por sus culturas y derechos políticos (Sturm, 1996: 119; Igor Q'aq'awitz Xoyon, com. pers., 2013; Rodríguez Guaján, com. pers., 2013). El uso de los glifos para crear estelas modernas como la de Iximche' cuenta como expresión creativa de un vínculo del pasado precolombino con la dinámica identidad panmaya del presente, que sigue creciendo en las comunidades mayas.

\section{Un estudio de caso: la estela de Iximche'}

El sitio de Iximche' se encuentra en el área central del altiplano guatemalteco, en la región kaqchikel, aproximadamente a $90 \mathrm{~km}$ al este de la ciudad de Guatemala y cerca de tres km al sur de Tecpán (Kraemer, 2007: 69). Destaca por haber sido el centro político precolombino de los kaqchikeles durante más de 50 años, antes de que los españoles la conquistaran y destruyeran en 1524 (Guillemin, 1967: 23-25). Bajo la dirección de George F. Guillemin se excavó el sitio entre 1958 y 1972; tras su fallecimiento, otros investigadores, como Stephen Whittington y Roger y Vally Nance se encargaron del análisis de los datos (Kraemer, 2007: 77). Hoy en día es un lugar turístico con un pequeño museo (figura 1). Es necesario destacar que en Iximche' no se ha encontrado evidencia de texto jeroglífico precolombino alguno (Grube y Fahsen, 2002: 233), y todavía se sigue discutiendo, si los kaqchikeles usaron la escritura jeroglífica en las épocas precolombinas, porque tampoco se ha encontrado evidencia material de ello (Christenson, 2001: 17; Sturm, 1996: 119).

En la entrada al sitio se erigió la estela de Iximche' el 21 de diciembre de 2012 para conmemorar el fin del $13^{\circ}$ bak'tun (figura 2; Méndez Villaseñor, 2012; Rodríguez, 2012; Rosales, 2012). Según el patrón que se ha establecido en la revitalización de las lenguas modernas, los expertos del pueblo kaqchikel que llevaron a cabo este trabajo consultaron con especialistas extranjeros, como el lingüista estadounidense Danny Law, pero a fin de cuentas fueron ellos quienes tomaron las decisiones creativas (Igor Q'aq'awitz Xoyon, com. pers., 2013; Rodríguez Guaján, com. pers., 2013). La estela surgió del trabajo colectivo de un equipo formado aproximadamente por 15 linguiistas, epigrafistas, historiadores y artistas, cuyos 


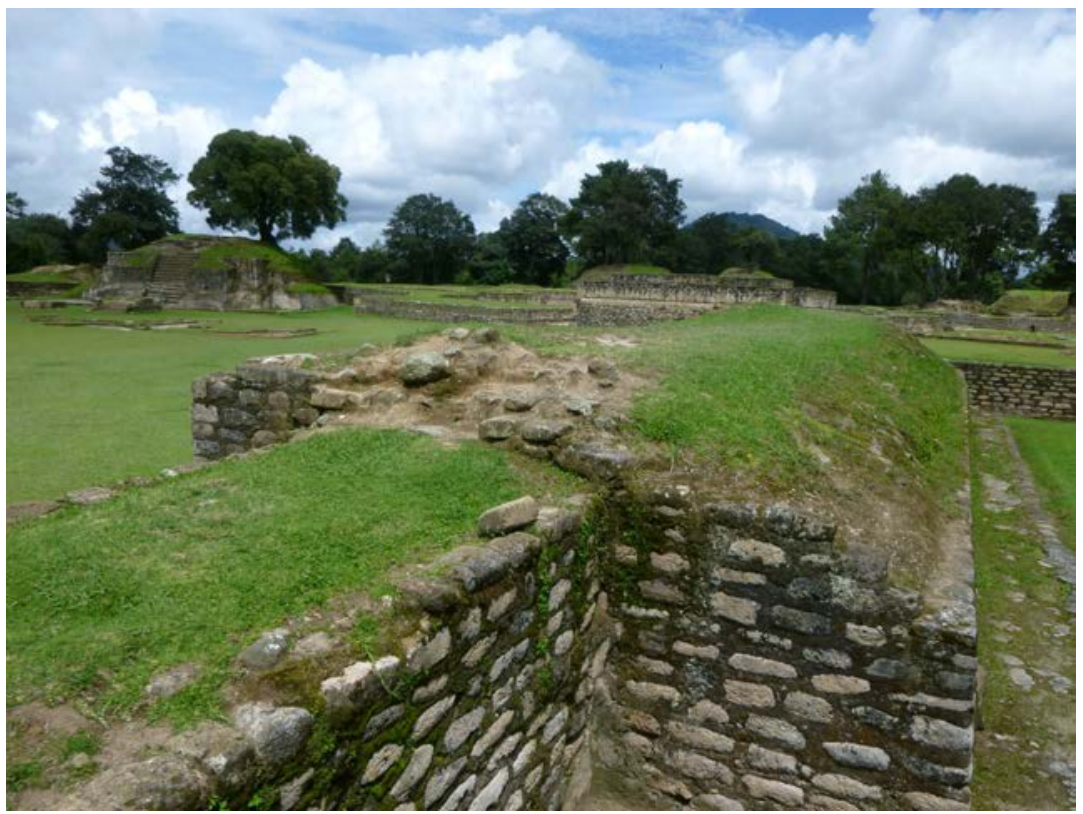

FIGURA 1. El sitio arqueológico de Iximche', Chimatenango, Guatemala

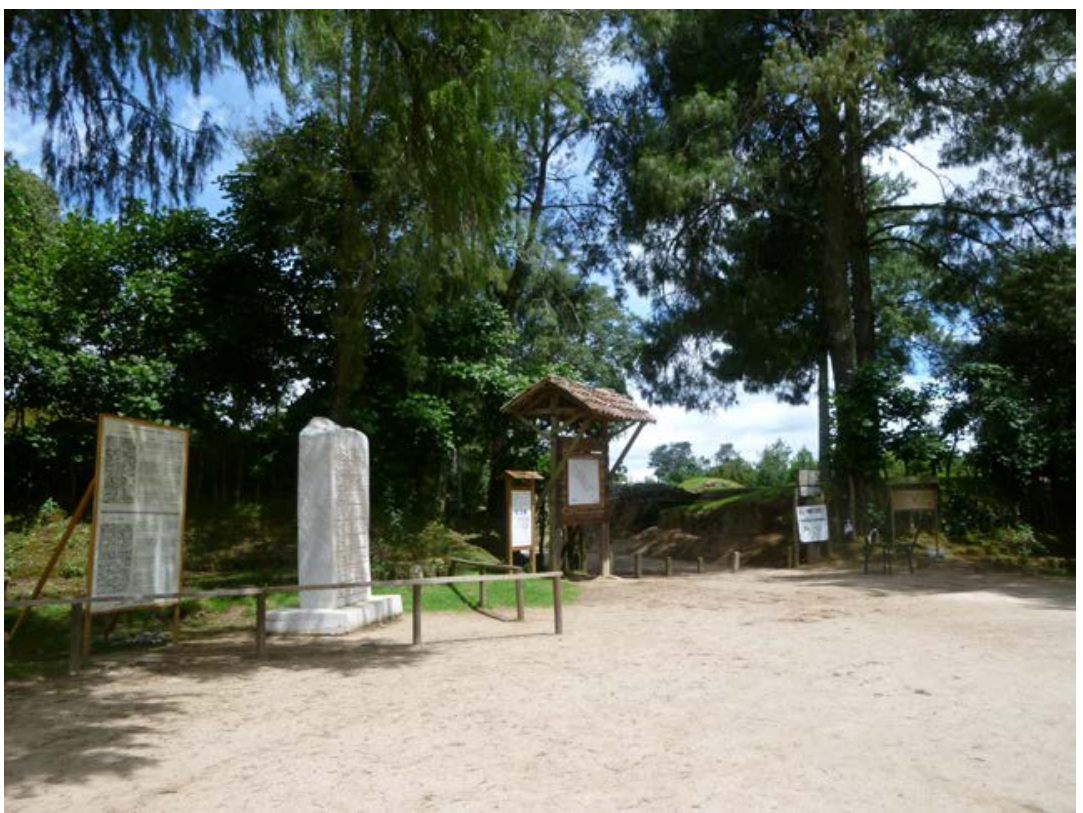

FiguRA 2. Estela moderna y placa con los dibujos, la transcripción y la traducción del texto en la entrada del sitio arqueológico de Iximche' 
miembros incluían al linguiista Pakal B’alam José Obispo Rodríguez Guaján, al educador Raxche' Demetrio Rodríguez Guaján, a los epigrafistas Igor Q’aq’awitz Xoyon e Iyaxel Cojtí Ren, al escultor Emerson Chicol, y a otros miembros de los grupos Sak Chuwen, ${ }^{8}$ y Kaqchikel Winäq Kaji' Imox ${ }^{9}$ (Igor Q'aq'awitz Xoyon, com. pers., 2013; Rodríguez Guaján, com. pers., 2013 y 2014; Sak Chuwen, 2013). ${ }^{10}$

El objetivo de los participantes no era sólo celebrar el fin del $13^{\circ}$ bak'tun, sino también realizar esta celebración a la manera de sus antepasados: "[S]erviría para conectarnos con nuestros antepasados y para darle valor a los tremendos sacrificios que ellos hicieron para que nosotros pudiéramos estar aquí el día de hoy" (Igor Q'aq'awitz Xoyon, com. pers., 2013). La ceremonia para festejar el fin del $13^{\circ}$ bak'tun duró un día y medio, e incluyó, entre otros eventos, la demostración del juego de pelota, bailes tradicionales, y la erección de la estela sobre una ofrenda de semillas de maíz, piedras y documentos en la base del monumento (Maxwell, 2012; Rosales, 2012). Además, la elección de Iximche' como lugar para eregir una estela moderna, implica la recuperación de su centro político histórico, destruido y abandonado a consecuencia de la conquista española. Los participantes recuperaron el pasado precolombino, no sólo la tradición de erigir estelas y la escritura jeroglífica, sino también su pertenencia al asentamiento de sus antepasados. Así, afirmaron y apoyaron un vínculo cultural y sociopolítico entre el presente y el pasado kaqchikeles.

En las dos superficies anchas de la estela, se tallaron 40 bloques glíficos que narran, en una forma histórica del idioma kaqchikel, ocho eventos destacados de la historia de los kaqchikeles (figuras 3-4). En la base y en los dos lados angostos de la estela se encuentra ilustrada la imagen de una estera (figura 5), la cual se describe en la literatura como símbolo precolombino del poder político (Robicsek, 1975; Schele y Miller, 1992: 71; Tate, 1992: 120). Al lado de la estela se colocó una placa con un dibujo del texto jeroglífico, donde figuran la transcripción del texto al kaqchikel y su traducción al castellano (figura 2; cuadro 1), para que los contenidos del texto lleguen a una audiencia más amplia. Sin embargo, en la placa aparecen expresiones diferentes para dirigirse a los kaqchikel hablantes y a los no hablantes respectivamente. Por ejemplo, se hace referencia a los bloques glíficos que corresponden a las frases transliteradas, pero sólo en la versión kaqchikel, y no en la traducción al castellano. Además, el texto jeroglífico y la transcripción en kaqchikel contienen la frase oj Maya' winäq, "nosotros

\footnotetext{
${ }^{8}$ El grupo Sak Chuwen se fundó en el año 2010 "con el fin de compartir el conocimiento ancestral de la antigua escritura y cultura de nuestros abuelos con sus descendientes, hijos todos de una gloriosa historia", por ejemplo a través de talleres de epigrafía (Sak Chuwen, 2012).

${ }^{9}$ Esta organización "se enfoca a la reividicación de los derechos del pueblo maya kaqchikel" (Igor Q'aq'awitz Xoyon, com. pers., 2013).

${ }^{10} \mathrm{El}$ autor del texto kaqchikel es Pakal Balam Rodríguez Guaján, quien también realizó el primer diseño del texto jeroglífico. Q'aq‘awitz Igor Xoyon, por su parte, mejoró el estilo y el diseño del texto, que fue revisado por Iyaxel Cojtí Ren (Rodríguez Guaján, com. pers., 2014). Los glifos fueron tallados por Emerson Chicol (Maxwell, 2012). El proyecto se realizó con el apoyo económico de, entre otros, la comunidad kaqchikel misma (Xoyon, com. pers., 2013).
} 
el pueblo maya", lo cual se traduce al castellano como "el Pueblo Maya", sin el pronombre en primera persona del plural, usado para indicar un sentimiento de pertenencia o unidad.
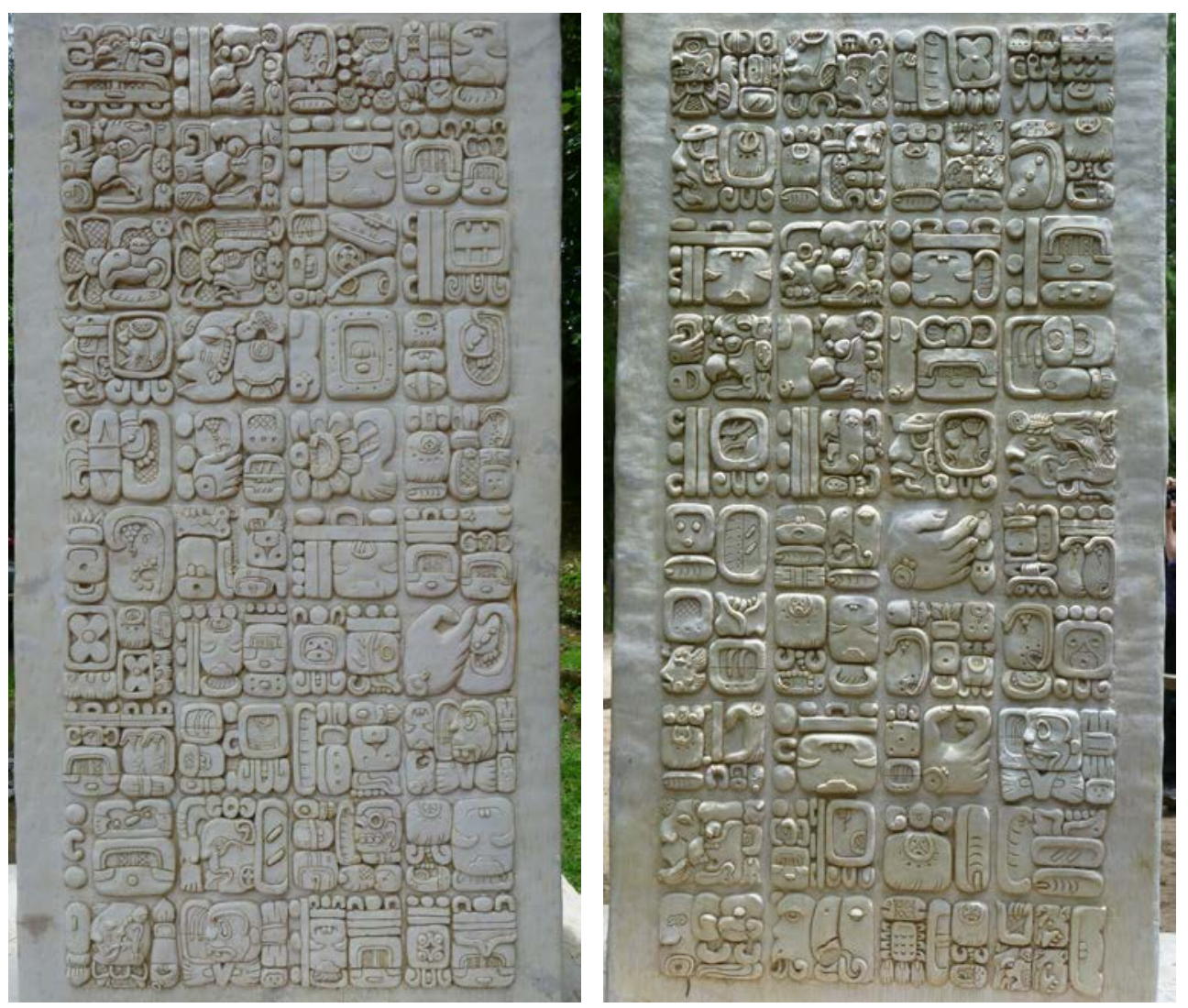

FIgURAS 3 y 4 . Los primeros 40 y los últimos 40 bloques del texto jeroglífico de la estela de Iximche'

Para la comunidad kaqchikel, el contenido de la estela "expresa nuestro pesar y nuestra esperanza como un pueblo en defensa de su idioma, cultura, escritura, cosmovisión, historia, etc." (Rodríguez Guaján, com. pers., 2013). El texto, como puede verse, inicia con la fecha en la forma típica de los consignados en las estelas clásicas, con la cuenta larga de 13.0.0.0.011 y la rueda calendárica kaqchikel

${ }^{11} \mathrm{Al}$ escribir la primera fecha así, los autores se decidieron por usarla como fecha base del ciclo calendárico actual, como propone Thompson (1950:149) (p. ej. Martin y Skidmore, 2012), aunque esta alternativa no es universalmente aceptada, y esta fecha también se escribe con frecuencia como 0.0.0.0.0 (Rice, 2007: 172). 


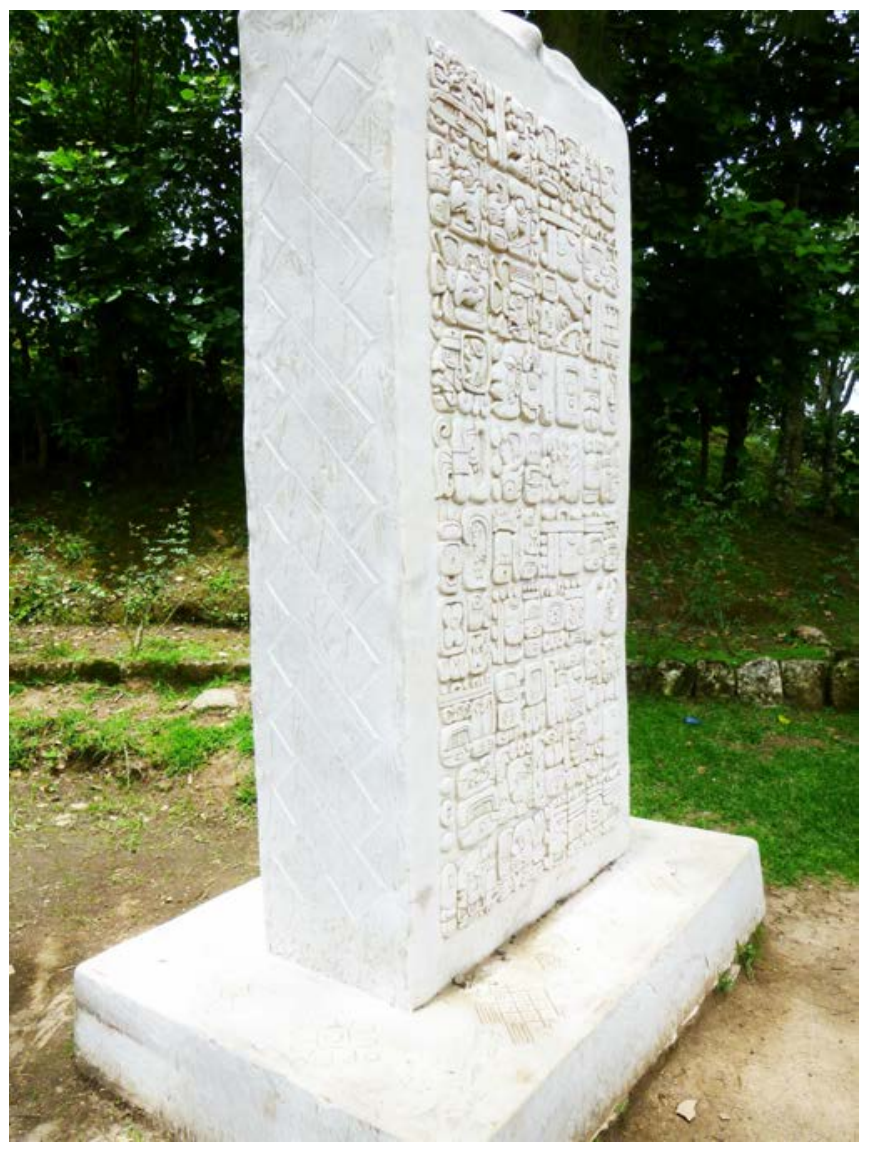

FiguRA 5. La estela de Iximche' con vista del diseño de estera tallado en los lados y en la base

de 4 Ajpu' 8 Kumk'u', y marca de esta manera la última creación del mundo y el comienzo del ciclo calendárico actual el 11 de agosto de 3114 a.C. ${ }^{12}$ El primer número de distancia lleva al lector de la fecha inicial a 11.12.9.13.4, 2 K'at 2 Wayeb' (el 9 de agosto de 470 d.C.), al día de la fundación de Iximche' por cuatro líderes del pueblo kaqchikel. Después se cuentan consecutivamente seis eventos más de la historia de los kaqchikeles: la rebelión entre los kaqchikeles bajo los Tuquche' en 11.13.11.7.13 $11 \mathrm{Aj} 1 \mathrm{Mol}$ (el 26 de diciembre de 1491); la llegada de los españoles a Iximche' en 11.15.4.4.0 1 Ajpu' 8 K'ank'in (el 24 de abril de 1524);

12 En la traducción al castellano en la placa se traduce la primera fecha en el calendario gregoriano como el 13 de agosto de 3114 a.C., pero es la única en la traducción que parece pertenecer a la correlación calendárica GMT (Goodman-Martínez-Thompson) de 584285, mientras que todas las demás corresponden a la correlación GMT de 584283 (véase Thompson, 1927). 
CuAdro 1. TRANSCRIPCIÓN KAQCHIKEL" Y TRADUCCIÓN AL CASTELLANO DEL TEXTO DE LA ESTELA

DE IXIMCHE' QUE SE ENCUENTRAN AL LADO DE ÉSTA, EN LA ENTRADA DEL SITIO ARQUEOLÓGICO

\begin{tabular}{|c|c|}
\hline Texto en kaqchikel & Texto en castellano \\
\hline $\begin{array}{l}\text { Chupam Kumk'u' oxlajpiq [oxlajb'ak'tun] chi } \\
\text { Kaji' Ajpu Waqxaqi' Kumk'u' xyatäj ox’ab’äj } \\
\text { pa kaj. Xtz'aq cholöy ajiläy q'ij mayq'ij. }\end{array}$ & $\begin{array}{l}\text { Guiado por K'ab'awil Kumk'u', en la cuenta } \\
\text { Oxlajpiq [Oxlajb'ak'tun] [13.0.0.0.0], el día } 4 \\
\text { Ajpu } 8 \text { Kumk'u' [13 de agosto del año } 3114 \\
\text { antes de la presente era] fueron colocadas } \\
\text { tres piedras de creación en el cielo y fueron } \\
\text { creados los contadores de la cuenta vigesi- } \\
\text { mal del tiempo. }\end{array}$ \\
\hline $\begin{array}{l}\text { Kajlej oxlajwinäq b'eleje' juna' [b'elejtun] } \\
\text { kab'laj winäq juna' [kab'lajtun] julajpiq } \\
\text { [julajb'ak'tun] k'a ri' chi Ka'i' K'at Ka'i' } \\
\text { Wayeb' xtz'uyub'äx tinamït Chi Iximche' } \\
\text { kuma kaji' kajawal Kaqchekel winäq. }\end{array}$ & $\begin{array}{l}4,649 \text { años tun y } 264 \text { días después } \\
\text { [11.12.9.13.4], el } 2 \text { K'at } 2 \text { Wayeb' fue fun- } \\
\text { dada la ciudad Chi Iximche' por cuatro ajaw } \\
\text { de los kaqchikel winäq [9 de agosto del año } \\
1470 \text { de la presente era]. }\end{array}$ \\
\hline $\begin{array}{l}\text { B'elejej kab’lajwinäq juna' [tun] juwinäq } \\
\text { juna' [juk'atun] chik xpakatäj ruxe' kaj chi } \\
\text { Julajuj Aj Jun Mol xb'anatäj yujuj ruma tu- } \\
\text { quche'. }\end{array}$ & $\begin{array}{l}21 \text { años tun y } 249 \text { días después [1.1.12.9], } \\
\text { amanece el } 11 \mathrm{Aj}, 1 \mathrm{Mol} \text { en el cual se hizo } \\
\text { la revuelta interna causada por los Tuquche' } \\
\text { [ } 26 \text { de diciembre del año } 1491 \text { de la pre- } \\
\text { sente era]. }\end{array}$ \\
\hline $\begin{array}{l}\text { Wuqub’ix kajlajwinäq kab’laj juna' [kab'lajtun] } \\
\text { juwinäq juna' [juk'atun] k’a ri chi Jun Ajpu } \\
\text { Waqxaqi' K'ank'in xulqaj Kastilan winäq Chi } \\
\text { Iximche'. Xtikïr qakamik oj Maya' winäq. }\end{array}$ & $\begin{array}{l}32 \text { años tun y } 287 \text { días después [1.12.14.7], } \\
\text { el } 1 \text { Ajpu } 8 \text { K'ank'in arribaron los españoles } \\
\text { a Chi Iximche' e inició la muerte del pue- } \\
\text { blo maya [ } 24 \text { de abril del año } 1524 \text { de la } \\
\text { presente era]. }\end{array}$ \\
\hline $\begin{array}{l}\text { Waqxaqej waqwinäq waqlajwinäq juna' } \\
\text { [waqlajk'atun] k'a ri, chi Oxlajuj Q'anel } \\
\text { Waqlajuj Pax xkamisäx xjitz'äx Ajaw } \\
\text { Ajposotz'il Kaji' Imox ruma Tunatiw. }\end{array}$ & $\begin{array}{l}16 \text { años tun y } 128 \text { días después [16.6.8], el } \\
13 \text { Q'anel } 16 \text { Pax murió ahorcado el ajaw } \\
\text { Ajposotz'il Kaji' Imox por Tunatiw [7 de ju- } \\
\text { nio del año 1541]. }\end{array}$ \\
\hline $\begin{array}{l}\text { Wuqub'ix kab'lajwinäq lajjuna' [lajtun] } \\
<\text { meWinaq juna'> jupiq [jub'ak'tun] k'a } \\
\text { ri' chi Julajuj Tz'kin Waqxaqlajuj K'ayab' } \\
\text { xelesäx teleche'al pa qawi' öj Maya' winäq } \\
\text { rumal Arewalo. }\end{array}$ & $\begin{array}{l}410 \text { años tun y } 247 \text { días después [1.0.10.12.7], } \\
\text { el } 11 \text { Tz'ikin } 18 \text { K'ayab' fue abolida la esclavi- } \\
\text { tud sobre los hombros del pueblo maya por } \\
\text { Arévalo [23 de marzo del año 1945]. }\end{array}$ \\
\hline $\begin{array}{l}\text { Kab'ij wuqwinäq <mejuna'> kajuna' } \\
\text { [k'atun] k'a ri' chi Waqi' No'j Wa'ix Muwan } \\
\text { xya' jub'a' qaq'ij pa Taqanem Wuj ruma } \\
\text { yojch'ojin öj maya'. }\end{array}$ & $\begin{array}{l}40 \text { años tun y } 142 \text { días después [2.0.7.2], } \\
\text { el } 6 \text { No’j } 0 \text { Muwan logramos que se nos re- } \\
\text { conociera en la Constitución Política de la } \\
\text { República [14 de enero del año 1985]. }\end{array}$ \\
\hline $\begin{array}{l}\text { Oxij waqwinäq waqxaqtun juk'atun }(1.8 ; 6.3) \\
\text { k'a ri' chi Kaji' Ajpu Oxi' K'ank'in xel xt- } \\
\text { zutzïr xtz'aqät oxlajpiq. Xtik tz'aläm ab'aj } \\
\text { Kaji' Ajpu rub'i' Chi Iximche'. Ruma qawixal } \\
\text { qonojel Maya' yojch'ojin. }\end{array}$ & $\begin{array}{l}28 \text { años tun y } 123 \text { días después [1.8.6.3], } \\
\text { el } 4 \text { Ajpu } 3 \text { K'ank'in se completa y termi- } \\
\text { na el Roxlajpiq [13.0.00.0], por ello en Chi } \\
\text { Iximche', se planta la estela [tz'aläm ab'äj] } \\
\text { llamada Kaji' Ajpu [21 de diciembre del año } \\
\text { 2012]. Por nuestras raíces, todos los mayas } \\
\text { seguimos revindicando, demandando. }\end{array}$ \\
\hline
\end{tabular}

* Véase nota anterior. 
el asesinato del líder kaqchikel Ajaw Ajposotz'il Kaji’ Imox por el conquistador Pedro de Alvarado en 11.16.0.10.8 13 Q'anil 16 Pax (el 7 de junio de 1540); la liberación del pueblo maya por el presidente guatemalteco Arévalo en 12.16.11.4.15 11 Tz'ikin 18 K'ayab' (el 23 de marzo de 1945); el reconocimiento del pueblo maya en el artículo 66 de la Constitución guatemalteca en 12.18.11.11.17 6 No’j 0 Muwan (el 14 de enero de 1985); y la erección de la estela de Iximche' y el fin del $13^{\circ}$ bak'tun en 13.0.0.0.0 4 Ajpu' $3 K^{\prime}$ 'ank'in (el 21 de diciembre de 2012).

Este artículo propone que el texto jeroglífico de la estela de Iximche' ilustra y contribuye en el proceso del resurgimiento cultural, linguiístico y jeroglífico de las comunidades mayas y del pueblo maya contemporáneos. Se usan glifos modificados para indicar sonidos que no aparecen en el corpus antiguo, logogramas sin y con complementos fonéticos, y una forma histórica del idioma kaqchikel de la época colonial. Es por ello, que el texto de Iximche' refleja la adaptación de la escritura al presente, la ideología de formas históricas en revalorar la herencia linguiística, y la negociación entre las identidades locales y la ideología de una identidad panmaya. Además, el texto manifiesta varios procesos, a veces en conflicto, de la creación de una identidad panmaya, primero por emplear glifos de una escritura antigua actualmente asociada con el concepto de un pasado común, también por estar escrito en una forma histórica del idioma kaqchikel, y finalmente por adaptar la escritura jeroglífica recuperada al contexto del presente. El estudio de éste y otros textos jeroglíficos modernos nos puede ayudar a entender los procesos de recuperación de una escritura olvidada, y también el contexto, las expresiones y el desarrollo del movimiento maya en su transformación linguiística, cultural y jeroglífica.

\section{La adaptación de la escritura al contexto linguiístico}

del presente en la estela de Iximche'

Uno de los procesos más visibles en el texto en la estela de Iximche' es la adaptación de la escritura jeroglífica antigua al contexto actual, tanto linguiístico como histórico, cultural y sociopolítico, que constituye un proceso fundamental al desarrollo de cada sistema de escritura (Schultz y Fecho, 2000) y a la revitalización o la recuperación linguiística, como se señaló antes. Este fenómeno también se puede observar entre los escribas modernos y otros expertos que recuperan la escritura jeroglífica. Tomando en cuenta el contexto del movimiento maya y la revitalización linguiística de las lenguas habladas, era de esperar que un componente fundamental para la recuperación de la escritura jeroglífica haya sido la adaptación de la escritura a los idiomas contemporáneos. El texto en la estela de Iximche' se escribió con glifos antiguos, pero en una forma histórica del kaqchikel conocido en textos de la época colonial (Xoyon, com. pers. 2013; Rodríguez Guaján, com. pers., 2013; p. ej. véase Maxwell y Hill, 2006), no en el idioma cholano en que se escribía la mayor parte de los textos jeroglíficos precolombi- 
nos, propio de otra rama de la familia de lenguas mayas (Campbell y Kaufman, 1985:194; Houston, Robertson y Stuart, 2000, 2001; Mora-Marín, 2003b, 2009; Robertson, 2010).

Como la estructura silábica prevaleciente de CVC (consonante-vocal-consonante) y el orden básico de constituyentes oracionales VOS (verbo-objeto-sujeto) en los idiomas mayas modernos corresponde a la que también se encuentra en la escritura jeroglífica (Campbell y Kaufman, 1985: 193; Sak Chuwen, 2011), ${ }^{13}$ la utilización de los glifos antiguos para escribir los idiomas modernos como el kaqchikel es, en principio, practicable. La mayor parte de las sílabas en el kaqchikel colonial y actual se encuentra también entre los glifos silábicos precolombinos (Johnson, 2013: fig. 1.14; Brown et al., 2006: tabla 1.2), pero para poder escribir precisamente en kaqchikel y otras lenguas modernas con los glifos antiguos, era necesario introducir nuevos glifos al corpus jeroglífico que representaran tonos que se usan actualmente, pero no se conocen en los textos precolombinos. Las consonantes fonémicas del kaqchikel colonial y actual que no se encuentran en el corpus jeroglífico precolombino son la oclusiva uvular sorda /q/, la oclusiva uvular glotalizada /q'/ y la vibrante múltiple alveolar/r/. Para escribir sílabas con estas consonantes como sonido inicial, se modificaron silabogramas precolombinos, agregándoles un signo diacrítico que asemeja la letra $<\mathrm{m}>$ de forma cursiva $o$, como en la estela de Iximche', la forma minúscula de la letra griega gamma $\langle\gamma\rangle$, dependiendo del escriba (Schele y Grube, 1996: 133; Sturm, 1996: 119-120; Sak Chuwen, 2011). Para crear las sílabas con/r/ inicial, se integró el diacrítico en silabogramas precolombinos con /y/ inicial; para las sílabas con /q/ y /q'/ iniciales, se usa como base los silabogramas con las consonantes iniciales $/ \mathrm{k} / \mathrm{y} / \mathrm{k}^{\prime} /$, respectivamente (Sak Chuwen, 2011). Por ejemplo, para escribir la primera sílaba de la frase "su nombre" en el texto de Iximche', se le agregó el diacrítico al silabograma antiguo $\mathrm{T} 61 / 62^{14}$ yu en una frase con b'i-i para indicar la pronunciación kaqchikel rub'i' (figura 6). Se supone que se escogieron estos signos como diacríticos porque no se asemejan a ningún signo en el corpus jeroglífico precolombino y de esta manera no se confunden con glifos antiguos.

Mientras que se ha adaptado la escritura jeroglífica a las lenguas modernas a fin de establecer nuevos glifos para los consonantes que no aparecen en el corpus precolombino, parece que no se ha intentado distinguir entre las vocales tensas y las vocales relajadas del kaqchikel en el texto de Iximche'. Tanto el kaqchikel estándar de hoy como el kaqchikel histórico de la época colonial tienen cinco vocales tensas $(/ \mathrm{a} /<\mathrm{a}\rangle, / \mathrm{e} /<\mathrm{e}\rangle, / \mathrm{i} /\langle\mathrm{i}\rangle, / \mathrm{o} /<\mathrm{o}\rangle, / \mathrm{u} /<\mathrm{u}\rangle)$ y cinco vocales relajadas $(/ \mathrm{i} /<\ddot{\mathrm{a}}>, / \varepsilon /<\ddot{\mathrm{e}}>, / \mathrm{I} /<\ddot{\mathrm{i}}>, / \mathrm{o} /<\ddot{\mathrm{o}}>, / \mathrm{v} /<\ddot{\mathrm{u}}>)$ como fonemas (Patal Majzul et al., 2000: 24; p. ej. is, "vello", vs. is, "papa"; öj, "nosotros" (pronombre absolutivo), vs. oj, "aguacate"). En el texto de Iximche', se escriben todas de la misma manera,

\footnotetext{
13 Para el kaqchikel actual véase Rodríguez Guaján, 1994: 32-33.

14 Para hacer referencia a glifos precolombinos específicos, se emplea el sistema establecido por Thompson (1962) . Véase también Montgomery, 2002: 12-13.
} 


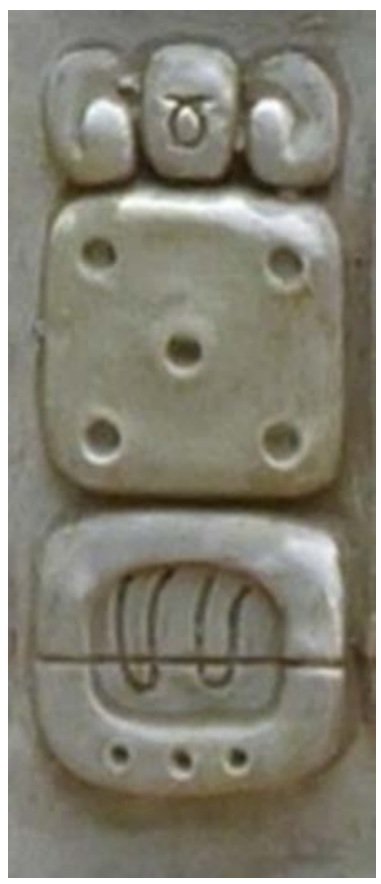

FIGURA 6. Ru-b’i-i rub'i' (“su nombre”) en la primera mitad bloque glífico G8 en la estela de Iximche’

sin indicar diferencia alguna, ni por usar glifos innovadores ni por emplear reglas de armonía o desarmonía vocálica (Houston et al., 1998; Lacadena y Wichmann, 2004). Por ejemplo, se escribe tinamit, "ciudad", "pueblo" como ti-na-mi-ti (bloque glífico A10), con armonía vocálica entre las últimas dos sílabas, donde la palabra kaqchikel tiene la vocal relajada $<i i>$. Pero también se usa el mismo patrón de armonía vocálica en escribir rub'i', "su nombre", que tiene la vocal tensa final $<i>$. como ru-b'i-i $i$ (p. ej. bloque glífico G8). De la misma manera, las palabras kaqchikeles kaqchikel y qonojel, "todos nosotros", tienen ambas la vocal tensa final $<\mathrm{e}>$, pero se emplean dos variedades diferentes de la desarmonía vocálica al escribir las últimas dos sílabas con los jeroglíficos, -ke-la y -je-li (bloques glíficos D1 y G10), respectivamente.

No parece existir ningún patrón exacto para indicar la tensión de las vocales kaqchikeles en la estela. Las palabras en el texto no se distinguen sólo por la tensión de sus vocales, y quizá debido a ello se tomó la decisión de no diferenciar entre las vocales relajadas y tensas en el texto jeroglífico. Pero esta decisión también puede indicar la discusión dentro de la comunidad kaqchikel linguística sobre el estatus de las vocales relajadas en la revitalización y la estandarización del idioma contemporáneo. Todas las cinco vocales relajadas aparecen en el kaqchikel estándar, pero en la práctica, el uso de las vocales relajadas difiere en 
parte según la variante: algunas de ellas tienen solamente una vocal relajada, la $\mid \mathrm{i} /<\ddot{\mathrm{a}}>$, mientras que otras tienen entre dos y cinco (Patal Majzul et al., 2000: 34). La decisión de no establecer reglas para distinguir entre las vocales tensas y relajadas en el texto de Iximche', podría reflejar una resistencia a entrar en la discusión, no poco polémica, sobre cuáles vocales se deberían estandarizar en el idioma hablado del presente.

La negociación entre la identidad kaqchikel

y la identidad panmaya en la estela de Iximche'

En el texto de Iximche' no se encuentran solamente silabogramas, sino también logogramas del corpus glífico precolombino. Un logograma representa una palabra o un morfema, no sólo un sonido o significado linguístico, en contraste con los silabogramas, loc cuales representan sílabas sin significado específico o propio (Kettunen y Helmke, 2011: 18). Muchos de los glifos en la estela de Iximche' son silábicos, sobre todo en las frases que cuentan los acontecimientos en sí, pero también aparecen varios logogramas, sobre todo en contextos calendáricos. Se usan casi sólo logogramas para representar los números y los periodos temporales en la cuenta larga, las fechas de tzolk'in y haab' y los números de distancia. Además, se emplea una variedad amplia de formas para estos logogramas, como se ve por ejemplo en la alternación entre números de puntos y barras (7 y 12) y de variantes de cabeza (10); y entre la forma básica (T521, WINIK/WINAL en el corpus precolombino, Kettunen y Helmke, 2011: tabla XI) y la variante de cabezas (T1034, HAAB') de los periodos de 20 días y los de 360, respectivamente, en escribir los primeros dos bloques glíficos del quinto número de distancia (figura 7).

Los logogramas no calendáricos son menos frecuentes que los calendáricos en la estela de Iximche', posiblemente debido a que el texto contiene muchos conceptos sin logograma correspondiente en el corpus jeroglífico precolombino, como es el caso del topónimo para Iximche' (p. ej. bloque glífico B10), las designaciones para los pueblos kaqchikel y maya (p. ej. bloques glíficos D1 y D9 respectivamente) y la Constitución de Guatemala (véase bloque glífico H1). Sin embargo, sí aparecen logogramas antiguos para representar palabras kaqchikeles, por ejemplo winäq, "pueblo" (T521, WINIK/WINAL en el corpus precolombino; p. ej. bloque glífico D1), wuj, "libro" (T609, HUN; bloque glífico H1), ul, "llegar" (T220v, HUL; bloque glífico D7), y ab’äj, "piedra" (T528, TUUN; p. ej. bloque glífico B5). Estos y otros logogramas en el texto conectan la estela de Iximche' directamente con los textos precolombinos, gracias a la reproducción de logogramas antiguos en el texto moderno. Así, los logogramas ayudan a crear una identidad panmaya, simbolizando no sólo un significado linguístico, sino también un sistema de escritura e, igualmente, una misma cultura e historia, que comparten todas las comunidades mayas (Maxwell, 1996: 199-200). 


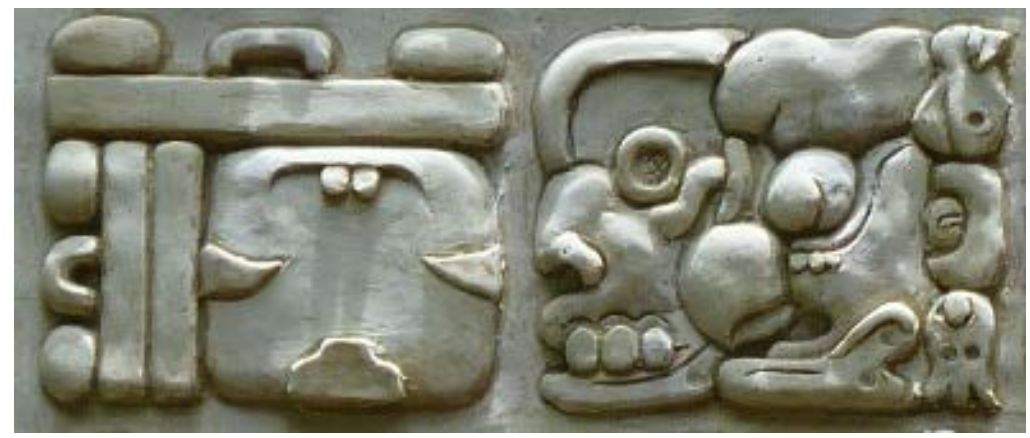

Figura 7. Los primeros dos bloques glíficos (E3-F3) de un número de distancia que muestran una alternación entre formas variantes de logogramas en la estela de Iximche'

Como los logogramas en los textos antiguos, los que se encuentran en el texto de Iximche', tienen la ventaja de que el lector puede entender su significado al reconocer la forma gráfica de los mismos sin depender de su lectura fonética y, por tanto, sin necesitar interpretarlos de manera literal o entender el kaqchikel hablado (Sturm, 1996: 121). Por sus posibilidades más amplias de comunicación, los logogramas en el texto de Iximche' asumen un valor simbólico, incluso para los lectores que no entienden el texto en un sentido linguiístico (Amery, 2001: 176-177; Sturm, 1996: 121).

El texto de Iximche' también afirma la identidad y el idioma de la comunidad kaqchikel como una unidad única dentro del pueblo maya, al combinar muchos, aunque no todos, los logogramas con complementos fonéticos (p. ej. T218 TZUTZ' que corresponde con kaqchikel xtzutzïr, "se termina”, bloque glífico G6). Para aclarar su pronunciación y así ayudar al lector a entender el texto, los escribas mayas antiguos solían añadir silabogramas como complementos fonéticos para los logogramas (Grube, 2010). Los mismos enfatizan de manera visual, que el idioma de la estela es el kaqchikel y no el maya clásico, además de cumplir con la función de indicar al lector la pronunciación kaqchikel del logograma.

Los complementos fonéticos aparecen con varios logogramas calendáricos para indicar su pronunciación en kaqchikel. Por ejemplo, el silabograma modificado qa con el glifo T521 "periodo de 20 días” (WINIK/WINAL en el corpus precolombino) señalando su pronunciación winäq en el quinto número de distancia (figura 8; véase también el uso de un mismo complemento fonético con el mismo logograma como la palabra kaqchikel winäq, "pueblo" en la segunda mitad del bloque glífico D1, figura 9). En cambio T521 WINIK/WINAL suele aparecer con los silabogramas ki o la como complemento fonético en los textos antiguos (p. ej. T521-ki en la plataforma del Templo XIX en Palenque, lado sur, bloque A3, véase Stuart, 2005: 191, 210; T521-la en el marcador del juego de pelota de Caracol, bloque B4, véase Helmke et al., 2006: fig. 2). Pero las combinaciones de logogramas con complementos fonéticos son más frecuentes en la estela moderna en contextos no 
calendáricos, sobre todo si la pronunciación kaqchikel del logograma no corresponde a su pronunciación precolombina. Si bien aparecen unas combinaciones en la estela de Iximche' que figuran en las inscripciones antiguas, como T1000d AJAW con complemento fonético T130 wa para indicar kaqchikel ajaw, "señor", en el bloque glífico $\mathrm{E} 1,{ }^{15}$ la mayoría de las combinaciones de logogramas con complementos fonéticos en la estela de Iximche' reflejan la identidad kaqchikel del texto. Por ejemplo, T609 'libro' (HUN en el corpus precolombino) combina con el complemento fonético T88 ji para indicar que corresponde con kaqchikel wuj (bloque glífico H1). De la misma manera, T736v 'muerte' (KAM/CHAM en el corpus precolombino) aparece con T102 ki para señalar la pronunciación de la sílaba final en kaqchikel kamik. Tales combinaciones de glifos no aparecían en los textos antiguos; surgieron como reacción a la necesidad de adaptar la escritura al idioma kaqchikel. Los logogramas indican la adaptación de la escritura jeroglífica recuperada al contexto del idioma kaqchikel en las combinaciones innovadoras de logogramas y complementos fonéticos que no aparecen en los monumentos jeroglíficos antiguos. Además, afirman la posición del idioma kaqchikel como parte de la identidad kaqchikel dentro del pueblo maya.

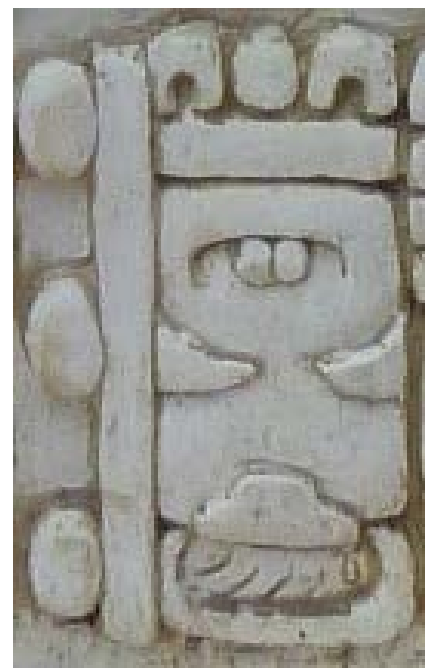

FIGURA 8. WINIK/WINAL-qa winäq (periodo de tiempo de 20 días) en la primera mitad del bloque glífico C10 en la estela de Iximche'

La ideología de las formas históricas en la estela de Iximche'

Al escribir el texto de la estela de Iximche' en kaqchikel, no en la lengua maya clásica del corpus jeroglífico precolombino, los creadores de la estela querían

\footnotetext{
${ }^{15}$ Véase un ejemplo de los textos precolombinos en Kettunen y Helmke, 2011, fig. 11.
} 
mantener y promover el valor del idioma kaqchikel y de la cultura de sus hablantes, reflejando pasivamente y cumpliendo activamente con estos objetivos del movimiento maya. No obstante, al reproducir una forma histórica del kaqchikel en lugar de su forma actual, el texto no sólo se asemeja más a la estructura de los textos jeroglíficos precolombinos (Rodríguez Guaján, com. pers., 2013), sino también afirma y apoya la identidad de las comunidades kaqchikeles de hoy como un pueblo unido por un mismo pasado, tanto linguí́stico como histórico (p. ej. Maxwell, 1996: 197). Tanto en el texto jeroglífico como en la revitalización de las lenguas contemporáneas ala que ya aludimos, el lenguaje histórico se usa para representar un pasado, en el que no existían tantas diferencias linguíísticas entre que los kaqchikel hablantes como hoy en día (England, 1996: 194, 2003: 739; French, 2010: 32-33; Maxwell, 1996: 199-200).

Por ser una forma anticuada del kaqchikel, el idioma del texto de Iximche' contiene varias formas que ya no se utilizan en el idioma actual. Una forma importante es la designación misma de kaqchikel, que aparece en el texto jeroglífico como la combinación del logograma T109 "rojo; gran" (CHAK en el corpus precolombino) con los silabogramas che-ke-la (figura 9), las vocales de la cual reflejan las de la forma kaqchekel que aparece junto con la forma actual kaqchikel en Las crónicas de los kaqchikeles (Maxwell y Hill, 2006: Parte II: 26). Además, la referencia a los conquistadores como los kastilan (ka-si-ti-la-ni; figura 10), señala la pronunciación de "castellano" en la época colonial (Maxwell y Hill, 2006: Parte II: 256), no la pronunciación kaxlan de hoy (Maxwell y Little, 2006: 17). Entre las formas históricas que no corresponden con las modernas, la más frecuente es la secuencia glífica ru-ma-le. Esta combinación de silabogramas indica la forma histórica rumal de la palabra kaqchikel contemporánea ruma o roma, una forma del sustantivo relacional -uma o -oma que en la estela indica la causa de una acción (p. ej. bloque glífico D5; véase la discusión anterior de -uma y -oma).

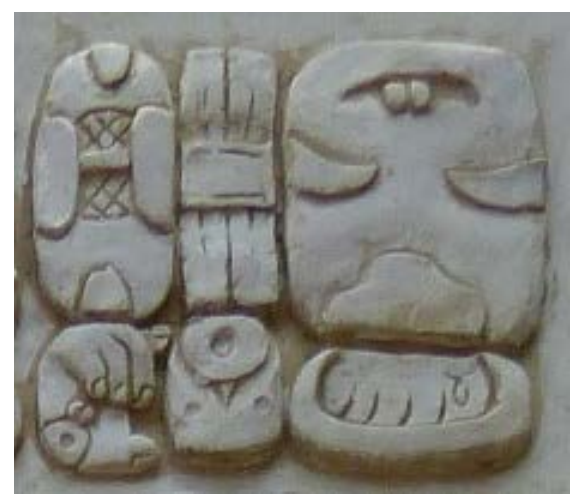

FIGURA 9. CHAK-che-ke-la WINIK/WINAL-qa Kaqchikel winäq ("pueblo kaqchikel”) en el bloque glífico D1 en la estela de Iximche' 


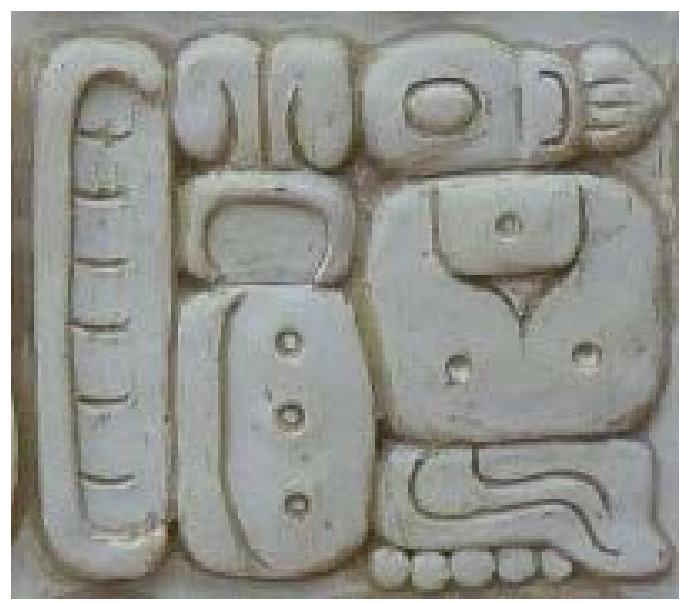

FIGURA 10. Ka-si-ti-la-ni kastilan (“castellano”) en el bloque glífico C8 en la estela de Iximche'

Ésta y las otras formas anticuadas sirven para enfatizar la herencia lingüística común de las variedades actuales del kaqchikel, queriendo representarlas como formas únicas y unidas del idioma histórico. Además de representar de forma escrita el pasado linguístico común de los kaqchikeles, el uso de su forma histórica evita el conflicto entre las variedades de hoy en la revitalización, sobre todo, como ya se apuntó, en lo que toca a la estandarización (p. ej. England, 2003: 738-740), por no elegir usar una forma específica, ya sea la forma estándar, ya otra variante actual, del kaqchikel moderno en el texto. De esta manera, se eligió usar una forma anticuada del idioma, la cual no se habla en ninguna comunidad actual, evitando privilegiar una variedad y marginar a los hablantes de las otras (p. ej. Maxwell, 1996: 205-206). Además, esta forma del kaqchikel se considera hoy en día como variedad representante histórica de sus hablantes como comunidad linguiística unida (Maxwell, 1996: 199). El texto también mantiene la unidad del pueblo kaqchikel, narrando en esta forma histórica una serie de hechos destacados en la historia de todos ellos, desde la última creación del mundo hasta la fundación de su centro político en Iximche' y la celebración del fin del $13^{\circ}$ bak'tun.

\section{Conclusión}

Los escribas modernos que crearon el texto jeroglífico de la estela de Iximche' participan de una tradición escribana moderna que se caracteriza por una fuerte influencia de origen en la investigación arqueológica, linguística y epigráfica; es decir, que el estudio y el conocimiento del sistema de escritura jeroglífico maya forman el esbozo reconstruido de la escritura recuperada. 
Los glifos mayas de hoy en día constituyen un sistema de escritura modernizado que se basa en el sistema precolombino, pero que, sin embargo, no es un descendiente directo de éste desarrollado como continuación de la tradición escribana precolombina. Dentro del esbozo reconstruido, la escritura sigue adaptándose y siendo adaptada al contexto linguístico, cultural y sociopolítico de las comunidades mayas y al movimiento maya (Brown, 1996: 174). Como parte de este proceso, se sigue extendiendo la escritura a contextos y medios nuevos (Grube y Fahsen, 2002: 233; p. ej. MAM, 2013a-c). Además, se ha modificado la escritura para representar las lenguas contemporáneas con más exactitud. La estela de Iximche' ilustra esta adaptación del sistema de escritura jeroglífico dentro del contexto de la revitalización linguística y resurgimiento cultural en el uso de nuevos glifos con un signo diacrítico y combinaciones innovadores de logogramas y complementos fonéticos para escribir el texto en una forma histórica del kaqchikel, en lugar de hacerlo en el idioma de los textos precolombinos.

La estela jeroglífica de Iximche' indica los procesos no solamente de adaptación de la escritura revitalizada a nuevos contextos, sino también aquellos relativos a negociar y redefinir la identidad, que también pertenecen a la revitalización linguística y al movimiento maya. Al examinar el texto se hacen evidentes las estrategias y los procesos de las comunidades mayas contemporáneas en retomar y redefinir su herencia cultural y linguística y aplicarla a nuevos contextos. Uno de los fenómenos más destacados es el uso del sistema de escritura jeroglífica, que representa una herencia cultural y linguística del pasado para todas las comunidades, para escribir un texto jeroglífico en kaqchikel. Este fenómeno refleja el diálogo entre el concepto reciente de una identidad panmaya y las identidades locales de las comunidades. La elección de una forma histórica del kaqchikel en lugar de una forma contemporánea refleja, reiteramos, no sólo las tensiones entre variedades locales en la revitalización del idioma, sino también los esfuerzos en unificar a los hablantes por representar una forma de la lengua que ya no caracteriza a ninguna comunidad o variedad específica, si no al pueblo kaqchikel como unidad linguiística, cultural e histórica.

La mencionada recuperación jeroglífica no justifica hablar de una equivocación o un uso erróneo de la escritura, como se ha insinuado (véase Brown, 1996: 174; Allen, 1992: 57, apud Brown, ibid.); se trata de un enriquecimiento general: a los miembros de las comunidades kaqchikeles y mayas se les abre un campo nuevo y expansivo para expresarse y desarrollarse, tanto en un sentido linguí́stico como cultural y sociopolítico, mientras que a los investigadores se les ofrece la oportunidad excepcional de estudiar el proceso extraordinario de recuperación de un sistema dormido de escritura.

Este ensayo propone, en consecuencia, que la investigación de la escritura jeroglífica maya moderna — no solamente la precolombina - goza de gran potencial para avanzar en la comprensión del papel que juegan los glifos mayas en los procesos de recuperación, así como el que desempeña la escritura en la reinterpretación y desarrollo de tradiciones e identidades en general. 


\section{Agradecimientos}

Deseo agradecer a Ixq'anil Judith M. Maxwell por compartir su conocimiento del idioma kaqchikel y por inspirar y apoyar los primeros pasos de este proyecto en el Tijonïk Kaqchikel Oxlajuj Aj. También a Pakal B’alam José Rodríguez Guaján, Q'aq'awitz Igor Xoyon y Demetrio Cojtí Cuxil por su entusiasmo y compartir sus conocimientos al responder a mis preguntas. iMatyöx, janila matyöx chiwe!

Agradezco asimismo a Martin Künne, Raina Heaton, y a los dos lectores anónimos sus valiosos comentarios sobre una primera versión de este trabajo. Y por último, pero no por eso menos importante, a Eileen Reich por su ayuda en la redacción del texto.

Este proyecto se realizó con el apoyo de becas de Summer Foreign Language Area Studies (Departamento de Educación de los Estados Unidos) y Fulbright (Comisiones de los Estados Unidos de América y Alemania). 


\section{BIBLIOGRAFÍA}

Allen, Arthur

1992 "Unriddling the glyphs: A new generation of Mayanists lets the Maya in on their secrets", Lingua Franca, pp. 52-58. New York: Academic Partners.

Amery, Rob

2001 “Language Planning and Language Revival”, Current Issues in Language Planning, 2 (2-3): 141-221.

Barrett, Rusty

2008 "Linguistic differentiation and Mayan language revitalization in Guatemala", Journal of Sociolinguistics, 12 (3): 275-305.

Bell, Jeanie

2013 "Language attitudes and language revival/survival", Journal of Multilingual and Multicultural Development, 34 (4): 399-410.

Brown, R. McKenna

1996 "The Mayan Language Loyalty Movement in Guatemala", Maya Cultural Activism in Guatemala, Edward F. Fischer y R. McKenna Brown (eds.), pp. 165177. Austin: University of Texas Press.

1998a "Mayan Language Revitalization in Guatemala", The Life of Our Language: Kaqchikel Maya Maintenance, Shift, and Revitalization, pp. 155-170, Susan Garzon, R. McKenna Brown, Julia Becker Richards y Wuqu' Ajpub’ (comps.). Austin: University of Texas Press.

1998b "A Brief Cultural History of the Guatemalan Highlands", The Life of Our Language: Kaqchikel Maya Maintenance, Shift, and Revitalization, pp. 44-61, Susan Garzon, R. McKenna Brown, Julia Becker Richards y Wuqu’ Ajpub’ (comps.). Austin: University of Texas Press.

Brown, R. McKenna, Judith M. Maxwell y Walter E. Little

2006 ¿La ütz awäch? Introduction to Kaqchikel Maya Language. Austin: University of Texas Press.

Campbell, Lyle y Terrence Kaufman

1985 "Mayan Linguistics: Where are we now?", Annual Review of Anthropology, 14: 187-198.

Cojtí Cuxil, Demetrio

1996 "The Politics of Maya Revindication”, Maya Cultural Activism in Guatemala, pp. 19-50, Edward F. Fischer y R. McKenna Brown (eds.). Austin: University of Texas Press.

2013 "Guatemala. La crisis y resiliencia entre los mayas tras el conflicto armado interno", ponencia presentada en la XVIII Conferencia Maya Europea. Bruselas, 1 de noviembre.

Chafe, Wallace L

1985 "Linguistic differences produced by differences between speaking and wri- 
ting", Literacy, Language, and Learning: The nature and consequences of reading and writing, pp. 105-123, David R. Olson, Nancy Torrance y Angela Hildyard (eds.). Cambridge: Cambridge University Press.

Christenson, Allen J.

2001 Art and Society in a Highland Maya Community: The Altarpiece of Santiago Atitlán. Austin: University of Texas Press.

Christie, Jessica Joyce (ed.)

2010 Maya Palaces and Elite Residences: An Interdisciplinary Approach. Austin: University of Texas Press.

Díaz-Andreu, Margarita y Sam Lucy

2005 "Introduction", The Archaeology of Identity: Approaches to Gender, Age, Status, Ethnicity and Religion, pp. 1-12, Margarita Díaz-Andreu, Sam Lucy, Staša Babić y Dadiv N. Edwards (eds.). New York: Routledge.

England, Nora

1996 "The Role of Language Standardization in Revitalization”, Maya Cultural Activism in Guatemala, pp. 178-194, Edward F. Fischer y R. McKenna Brown (eds.). Austin: University of Texas Press.

1998 "Mayan efforts toward language preservation”, Endangered Languages: Current Issues and Future Prospects, pp. 99-116, Lenore A. Grenoble y Lindsay J. Whaley (eds.). Cambridge: Cambridge University Press.

2002 "Maya Linguists, Linguistics, and the Politics of Identity", Texas Linguistic Forum, 45: 33-45.

2003 "Mayan Language Revival and Revitalization Politics: Linguists and Linguistic Ideologies", American Anthropologist, 105 (4): 733-743.

Fischer, Edward F.

1996 "Induced Cultural Change as a Strategy for Socioeconomic Development: The Pan-Maya Movement in Guatemala”, Maya Cultural Activism in Guatemala, pp. 51-73, Edward F. Fischer y R. McKenna Brown (eds.). Austin: University of Texas Press.

Fishman, Joshua A.

2011 "If Threatened Languages can be Saved, then can Dead Languages be Revived?", Current Issues in Language Planning, 2 (2-3): 222-230.

French, Brigittine M.

2010 Maya Ethnolinguistic Identity: Violence, Cultural Rights, and Modernity in Highland Guatemala. Tucson: The University of Arizona Press.

Grenoble, Lenore A.

2013 "Language Revitalization”, The Oxford Handbook of Sociolinguistics, pp. 792811, Robert Bayley, Richard Cameron y Ceil Lucas (eds.). Oxford: Oxford University Press. 
Grivelet, Stéphane

2001 "Digraphia in Mongolia", International Journal of the Sociology of Language 150: 75-93.

Grube, Nikolai

2010 "Preposed Phonetic Complements in Maya Hieroglyphic Writing", Linguistics and Archaeology in the Americas: The Historization of Language and Society, pp. 27-44, Eithne B. Carlin y Simon van de Kerke (eds.). Leiden: Koninklijke Brill.

Grube, Nikolai y Fredrico Fahsen

2002 "The Workshops on Maya History and Writing in Guatemala and México", Heart of Creation: The Mesoamerican World and the Legacy of Linda Schele, pp. 216-237, Andrea Stone (ed.). Tuscaloosa: University of Alabama Press.

Guillemin, George F.

1967 “The Ancient Cakchiquel Capital of Iximche”, Expedition, 9: 22-35.

Helmke, Christophe, Harri Kettunen y Stanley Guenter

2006 "Comments on the Hieroglyphic Texts of the B-Group Ballcourt Markers at Caracol, Belize", Wayeb Notes 23. European Association of Mayanists, <www. wayeb.org/notes/wayeb_notes0023.pdf $>$ [Último acceso 2 de julio de 2014].

Houston, Stephen

1994 "Literacy among the Pre-Columbian Maya: A Comparative Perspective", Writing Without Words: Alternative Literacies in Mesoamerica and the Andes, pp. 27-49, Elizabeth Hill Boone y Walter D. Mignolo (eds.). Durham: Duke University Press.

1998 (ed.) Function and Meaning in Classic Maya Architecture: A Symposium at Dumbarton Oaks, 7th and 8th October 1994. Washington, D. C.: Dumbarton Oaks.

2008 "The Small Deaths of Maya Writing", The Disappearance of Writing Systems: Perspectives on Literacy and Communication, pp. 231-252, John Baines, John Bennet y Stephen Houston (eds.). London: Equinox Publishing.

Houston, Stephen, David Stuart y John Robertson

1998 "Disharmony in Maya hieroglyphic writing: linguistic change and continuity in Classic society", Anatomía de una civilización: aproximaciones interdisciplinarias a la cultura maya, pp. 275-296, Andrés Ciudad Ruiz et al. (eds.). Madrid: Sociedad Española de Estudios Mayas (Publicaciones de la SEEM, 4).

Houston, Stephen, John Robertson y David Stuart

2000 "The Language of the Classic Maya Inscriptions", Current Anthropology, 41 (3): 321-356.

2001 "More on the Language of the Classic Maya Inscriptions", Current Anthropo$\log y, 42$ (4): 558-559.

Houston, Stephen, Oswaldo Chinchilla Mazariegos y David Stuart

2001 "Introduction", The Decipherment of Ancient Maya Writing, pp. 3-19, Stephen Houston, Oswaldo Chinchilla Mazariegos y David Stuart (eds.). Norman: University of Oklahoma Press. 
Jones, Mari C.

1998 Language Obsolescence and Revitalization: Linguistic Change in Two Sociolinguistically Contrasting Welsh Communities. Oxford: Oxford University Press.

Kettunen, Harri y Christophe Helmke

2011 Introducción a los jeroglíficos mayas. Copenhague: XVI Conferencia Maya Europea.

King, Kendall A.

2001 Language Revitalization Processes and Prospects: Quichua in the Ecuadorian Andes. Clevedon, Reino Unido: Multilingual Matters.

Kraemer, Anne E.

2007 "Unearthing Collaboration: Community and Multivocal Archaeology in Highland Guatemala", tesis de maestría en Antropología. Lawrence: University of Kansas.

Krochock, Ruth J.

2002 "Written Evidence", Handbook to Life in the Ancient Maya World, pp. 265-304, Lynn V. Foster (ed.). Oxford: Oxford University Press.

Lacadena, Alfonso

1995 "Revitalización de grafías escriturarias arcaicas en el Clásico Tardío maya", Religión y Sociedad en el área maya, pp. 29-41, Carmen Varela, Juan Luis Bonor y Yolanda Fernández (eds.). Madrid: Sociedad Española de Estudios Mayas, Instituto de Cooperación Iberoamericana y Caja de Madrid (Publicaciones de la SEEM, 3).

Lacadena, Alfonso y Søren Wichmann

2004 "On the Representation of the Glottal stop in Maya Writing", The Linguistics of Maya Writing, pp. 103-162, Søren Wichmann (ed.). Salt Lake City: The University of Utah Press.

Law, Danny

2013 "Mayan Historical Linguistics in a New Age", Language and Linguistics Compass, 7 (3): 141-156.

Macri, Martha J. y Gabrielle Vail

2009 The New Catalog of Maya Hieroglyphs, vol. 2: The Codical Texts. Norman: University of Oklahoma Press.

Macri, Martha J. y Matthew G. Looper

2003 The New Catalogue of Maya Hieroglyphs, vol. 1: The Classic Period Inscriptions. Norman: University of Oklahoma Press.

MAM (Maya Antiguo para los Mayas/Mayas for Ancient Mayan)

2013a "5 Ajaw 18 Yax (October 17, 2013): Glyphs on Pots", <http://discovermam. org/> [Último acceso 2 de enero de 2014]. 
2013b “13 Ajaw 18 Mak (December 16, 2013): Four more stelae”, <http://discovermam.org/> [Último acceso 2 de enero de 2014].

2013c "6 Ajaw 18 Kej (November 26, 2013): Two stelae, two Maya languages", $<$ http://discovermam.org/> [Último acceso 2 de enero de 2014].

2014 "3 Ajaw 13 Pop (April 15, 2014): Contemporary Maya poem, in glyphs!", $<$ http://discovermam.org/> [Último acceso 10 de junio de 2014].

Martin, Simon y Joel Skidmore

2012 "Exploring the 584286 Correlation between the Maya and European Calendars", The PARI Journal, 13 (2): 3-16.

Martin, Simon y Nikolai Grube

2008 Chronicles of the Maya Kings and Queens: Deciphering the Dynasties of the Ancient Maya, $2^{\mathrm{a}}$ ed. London: Thames and Hudson.

Matsumoto, Mallory

2013 "Reflection as Transformation: Mirror-Image Structure on Maya Monumental Texts as a Visual Metaphor for Ritual Participation", Estudios de Cultura Maya, XLI: 93-128.

Maxwell, Judith M.

1996 "Prescriptive Grammar and Kaqchikel Revitalization”, Maya Cultural Activism in Guatemala, pp. 195-207, Edward F. Fischer y R. McKenna Brown (eds.). Austin: University of Texas Press.

2012 "Oxlajuj B'aqtun / Oxlajun Pik The Dawning of the New Maya Era”, The Maxwell Blog, Tulane University School of Liberal Arts and Research, New Orleans, Louisiana, 21 de diciembre de 2012, < http://tulane.edu/liberal-arts/ blogs/maxwell/new-maya-era.cfm > [Último acceso 5 de junio de 2014].

Maxwell, Judith M. y Robert M. Hill II (trads. y eds.)

2006 The Kaqchikel Chronicles: The Definitive Edition. Austin: University of Texas Press.

Maxwell, Judith M. y Walter E. Little

2006 Tijonïk Kaqchikel Oxlajuj Aj: Curso de idioma y cultura maya kaqchikel. Antigua, Guatemala: Editorial Junajpu'.

Méndez Villaseñor, Claudia

2012 "La alborada del Oxlajuj Bak'tun”, El Periódico, Guatemala, 16 de diciembre de 2012, <http://www.elperiodico.com.gt/es/20121216/pais/222162/?tpl=54> [Último acceso 13 de enero de 2014].

Montejo, Víctor

2002 "The Multiplicity of Maya Voices", Indigenous Movements, Self-Representation, and the State in Latin America, pp. 123-148, Kay B. Warren y Jean E. Jackson (eds.). Austin: University of Texas Press.

2005 Maya Intellectual Renaissance: Identity, Representation, and Leadership. Austin: University of Texas Press. 
Montgomery, John

2002 Dictionary of Maya Hieroglyphs. New York: Hippocrene Books.

Mora-Marín, David

2003a "The origin of Mayan syllabograms and orthographic conventions", Written Language and Literacy, 6 (2): 193-238.

2003b "Pre-Ch'olan as the Standard Language of Classic Lowland Mayan Texts", ponencia en la 2002-2003 SSILA-Conferencia, Atlanta, Georgia, enero de 2003, <http://davidmm.web.unc.edu/files/2010/08/PreCholanSSILA.pdf > [Último acceso 1 de enero de 2014].

2009 "A Test and Falsification of the "Classic Ch'olti'an" Hypothesis: A Study of Three Proto-Ch'olan Markers”, International Journal of American Linguistics, 75 (2): 115-157.

Mucia Batz, José

2012 "Laqamtzununya", <https://sites.google.com/site/torneocha2012/laqamtz ununya $>$ [Último acceso 9 de enero de 2014].

Normark, Johan

2004 "Discontinuous Maya Identities-Culture and Ethnicity in Maya Discourse", Material Culture and Other Things, pp. 109-160, Frederik Fahlander y Terje Oestigaard (eds.). Gothenburg: University of Gothenburg.

Otzoy, Irma

1996 "Maya Clothing and Identity", Maya Cultural Activism in Guatemala, pp. 141155, Edward F. Fischer y R. McKenna Brown (eds.). Austin: University of Texas Press.

Patal Majzul, Filiberto, Pedro Oscar García Matzar y Carmelina Espantzay

2000 Rujunamaxik ri Kaqchikel Chi'. Variación dialectal en Kaqchikel. Guatemala: Editorial Cholsamaj.

Pouessel, Stéphanie

2008 "Écrire la langue berbère au royaume de Mohamed VI: Les enjeux politiques et identitaires du tifinagh au Maroc", Revue des Mondes Musulmans et de la Méditerranée, 124: 219-239.

Rice, Prudence M.

2007 Maya Calendar Origins: Monuments, Mythistory, and the Materialization of Time. Austin: University of Texas Press.

Robertson, John S.

2010 "From Common Cholan-Tzeltalan to Classical Ch'olti: The Identification of the Language of Mayan Hieroglyphs", Mesoweb, <http://www.mesoweb.com/articles/Robertson/Robertson-2010.pdf> [Último acceso 1 de enero de 2014].

Robicsek, Francis

1975 A Study in Maya Art and History: The Mat Symbol. New York: Museum of the American Indian, Heye Foundation. 
Rodríguez, Francisco

2012 "Kaqchikeles vuelen a tallar su historia. Dos estelas fueron mostradas para conmemorar la celebración del Bak'tun”, El Periódico, Guatemala, 22 de diciembre de 2012, <http://www.elperiodico.com.gt/es/20121222/ pais/222483/?tpl=>.

Rodríguez Guaján, José Obispo

1994 Rutz’ib’axik ri Kaqchikel. Manual de redacción kaqchikel. Guatemala: Editorial Cholsamaj.

Rosales, José

2012

"Develan estela maya en el centro arqueológico de Iximche”,, Prensa Libre, Guatemala, 21 de diciembre de 2012, <http://www.prensalibre.com/mayas_2012/Develan-estela-centro-arqueologico-Iximche_0_832716917.html> [Último acceso 5 de junio de 2014].

Sak Chuwen

2011 Apéndice, nivel introductorio. Guatemala: Centro de Investigación y Estudio de Epigrafía Maya Sak Chuwen.

2012 "Y empezamos a caminar...", Sak Chuwen, Guatemala, <http://sakchuwen. blogspot.de/> [Último acceso 5 de junio de 2014].

2013 "8 Ajaw 18 Sip (May 30, 2013): 2012 Report from Sak Chuwen", <http:// discovermam.org $>$ [Último acceso 13 de enero de 2014].

Scilar-Cabral, Leonor

2012 "Positive effects of Psycholinguistic and Neuroscience findings today", Applied Psycholinguistics: Positive effects and ethical perspectives, vol. 1, pp. 37-45. Giussepe Mininni y Amelia Manuti (eds.). Milano: Franco Angeli.

Schele, Linda

2000 "The Linda Schele Drawings Collection", < http://research.famsi.org/schele. html > [Último acceso 9 de enero de 2014].

Schele, Linda y Mary Ellen Miller

1992 The Blood of Kings: Dynasty and Ritual in Maya Art. New York: George Braziller y Kimball Art Museum.

Schele, Linda y Nikolai Grube

1996 "The Workshop for Maya on Hieroglyphic Writing", Maya Cultural Activism in Guatemala, pp. 131-140, Edward F. Fischer y R. McKenna Brown (eds.). Austin: University of Texas Press.

Schultz, Katherine y Bob Fecho

2000 "Society's Child: Social Context and Writing Development", Educational Psychologist, 35 (1): 51-62.

Spolsky, Bernard

1991 "Hebrew language vitalization within a general theory of second language learning", The Influence of Language on Culture and Thought: Essays in Honor 
of Joshua A. Fishman's Sixty-Fifth Birthday, pp. 137-156, Robert L. Cooper y Bernard Spolsky (eds.). New York: Mouton de Gruyter.

Steinberg, Danny D. y Natalia V. Sciarini

2006 An Introduction to Psycholinguistics, $2^{\mathrm{a}}$ ed. New York: Routledge.

Stuart, David

2005 The Inscriptions from Temple XIX at Palenque: A Commentary. San Francisco: The Pre-Columbian Art Research Institute.

Sturm, Circe

1996 "Old Writing and New Messages: The Role of Hieroglyphic Literacy in Maya Cultural Activism”, Maya Cultural Activism in Guatemala, pp. 114-130, Edward F. Fischer y R. McKenna Brown (eds.). Austin: University of Texas Press.

Tate, Carolyn Elaine

1992 Yaxchilán: The Design of a Maya Ceremonial City. Austin: University of Texas Press.

Thompson, J. Eric S.

1927 "A Correlation of the Maya and European Calendars", Field Museum of Natural History, Anthropological Series, 17 (1): 1-22.

1950 Maya Hieroglyphic Writing: Introduction. Washington, D. C.: Carnegie Institute of Washington.

1962 A Catalog of Maya Hieroglyphs. Norman: University of Oklahoma Press.

Tsunoda, Tasaku

2005 Language Endangerment and Language Revitalization: An Introduction. New York: Mouton de Gruyter.

Unseth, Peter

2005 "Sociolinguistic parallels between choosing scripts and languages", Written Language and Literacy, 8 (1): 19-42.

Warren, Paul

2013 Introducing Psycholinguistics. Cambridge: Cambridge University Press.

Wilson, Gary N.

2009 "But the Language Has Got Children Now. Language Revitalisation and Education Planning in the Isle of Man”, Shima: The International Journal of Research into Island Cultures, 3 (2): 15-31.

Zuckermann, Ghil'ad

2009 "Hybridity versus Revivability: Multiple Causation, Forms and Patterns", Journal of Language Contact VARIA, 2: 40-67.

Zuckermann, Ghil'ad y Michael Walsh

2011 "Stop, Revive, Survive: Lessons from the Hebrew Revival Applicable to the Reclamation, Maintenance and Empowerment of Aboriginal Languages and Cultures", Australian Journal of Linguistics, 31 (1): 111-127. 